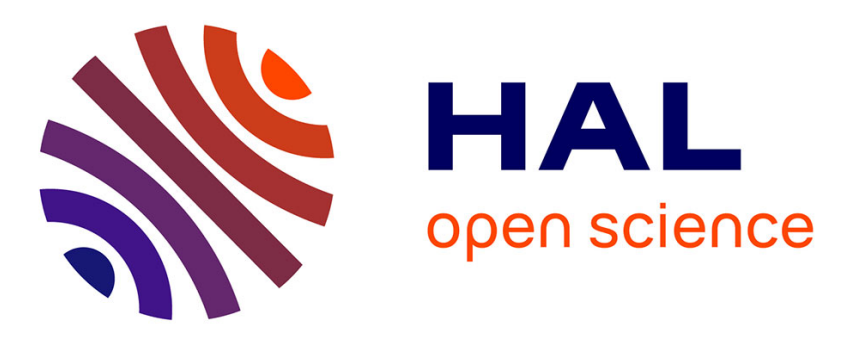

\title{
Elastic and electrical behavior of some random multiscale highly-contrasted composites
}

François Willot, Dominique Jeulin

\section{To cite this version:}

François Willot, Dominique Jeulin. Elastic and electrical behavior of some random multiscale highlycontrasted composites. International Journal for Multiscale Computational Engineering, 2011, 9 (3), pp.305-326. 10.1615/IntJMultCompEng.v9.i3.40 . hal-00553376

\section{HAL Id: hal-00553376 \\ https://hal-mines-paristech.archives-ouvertes.fr/hal-00553376}

Submitted on 7 Jan 2011

HAL is a multi-disciplinary open access archive for the deposit and dissemination of scientific research documents, whether they are published or not. The documents may come from teaching and research institutions in France or abroad, or from public or private research centers.
L'archive ouverte pluridisciplinaire HAL, est destinée au dépôt et à la diffusion de documents scientifiques de niveau recherche, publiés ou non, émanant des établissements d'enseignement et de recherche français ou étrangers, des laboratoires publics ou privés. 


\title{
ELASTIC AND ELECTRICAL BEHAVIOR OF SOME RANDOM MULTISCALE HIGHLY-CONTRASTED COMPOSITES
}

\author{
FRANÇOIS WILLOT* AND DOMINIQUE JEULIN ${ }^{\dagger}$
}

\begin{abstract}
The role of a non-uniform distribution of heterogeneities on the elastic as well as electrical properties of composites is studied numerically and compared with available theoretical results. Specifically, a random model made of embedded Boolean sets of spherical inclusions (see e.g. Jean et al, 2007) serves as the basis for building simple two-scales microstructures of "granular"type. Materials with "infinitely-contrasted" properties are considered, i.e. inclusions elastically behave as rigid particles or pores, or as perfectly-insulating or highly-conducting heterogeneities. The inclusion spatial dispersion is controlled by the ratio between the two characteristic lengths of the microstructure. The macroscopic behavior as well as the local response of composites are computed using full-field computations, carried out with the "Fast Fourier Transform" method (Moulinec and Suquet, 1994). The entire range of inclusion concentration, and dispersion ratios up to the separation of length scales are investigated.

As expected, the non-uniform dispersion of inhomogeneities in multi-scale microstructures leads to increased reinforcing or softening effects compared to the corresponding one-scale model (Willot and Jeulin, 2009); these effects are however still significantly far apart from Hashin-Shtrikman bounds. Similar conclusions are drawn regarding the electrical conductivity.
\end{abstract}

Key words. Homogenization, Linear elastic media, Linear conductors, Reinforced composites, Multiscale models, Porous media, Percolation, RVE

AMS subject classifications. 74B05, 74Q20, 74Q05

1. Introduction. In many practical situations, composite materials are made of a non homogeneous distribution of inclusions, as a result of a non uniform mixing during the dispersion of inclusions in the matrix. For instance, this is the case of carbon black nanoparticles in a resin matrix $(13 ; 41 ; 18 ; 5)$, in an elastomer matrix (10), or of carbon nanotubes (19), that are dispersed into clusters. Fortunately, this non homogeneous distribution is not detrimental to the macroscopic properties of composites in the case of reinforcement particles (e.g. more conductive or stiffer than the matrix). The object of this paper is to predict these effects for specific multiscale models, namely combinations of Boolean models of spheres in the case of a very high contrast of properties.

After a reminder of the construction and of some morphological properties of multiscale Cox Boolean models, the corresponding analytical third order bounds are recalled. For the high contrast case, the practical use of bounds to predict the effective properties is limited, and since no general analytical tools are available, it is required to have recourse to numerical techniques on simulated microstructures in order to estimate local fields and homogenized properties. In the present case we make use of FFT numerical estimates for conductivity and linear elasticity, implemented on simulated binary images of the microstructure. This numerical tool that does not need any meshing of the microstructure can handle rather large systems (up to $750^{3}$ in the present case), and is therefore well suited to complex multiscale microstructures. Our approach is applied to the study of the mechanical behavior of multiscale rigidlyreinforced or porous composites, as well as to the electrical behavior of multiscale composites with highly conducting or perfectly insulating inclusions. Finally, the

*MINES-ParisTech, Centre de Morphologie Mathématique, Mathématiques et Systèmes, 35 rue Saint Honoré, F-77305 Fontainebleau CEDEX, France (francois.willot@mines-paristech.fr.)

$\dagger$ idem, dominique.jeulin@mines-paristech.fr 
statistical fluctuations of fields observed in simulations are accounted for to define a statistical RVE, and to give a precision of our results.

2. Preliminaries on multiscale random media. Multiscale models of random media are of common practice in geostatistics, for instance in order to describe the complex distribution of minerals in orebody deposits (28). The most usual approach is to decompose a given random function $Z(x)$ into the sum of $n$ uncorrelated components $Z_{i}(x)$, with $Z(x)=\sum_{i=1}^{i=n} Z_{i}(x)$. For stationary random fucntions, this can be made through the decomposition of the central covariance of $Z(x)$ (see the definition in section 9.1) into the sum of models of covariances with different scales, as it is done in the mining area. For random sets, the addition has to be replaced by some set operations, like the union or the intersection of random sets with different scales (13). Other models exist, like hierarchical models built on random tesselations of space $(13 ; 16)$. In what follows, we will consider generalizations of the one scale Boolean model $(26 ; 29)$ to multiscale Boolean model based on Cox point processes, as proposed in (14).

\section{Multiscale random media.}

3.1. One-scale Boolean model. In the "one-scale model", noted (B), a Boolean random set of spheres $(26 ; 29)$ is used to generate microstructure samples. It is recalled that when inclusions are located on the Poisson points with a homogeneous intensity (average number of points per unit volume) $\theta$, the volume fraction of spheres $f$ is given by:

$$
f=1-\mathbf{e}^{-\theta \frac{4 \pi}{3} a^{3}}
$$

To account for microstructures variability in a bounded domain $\Omega$, the Boolean samples used in model (B) are made up of a varying number of spheres. The random number of centers of spheres contained in $\Omega$ (here a cube with edge $L$ ) follows a Poisson distribution with average $\lambda=\theta L^{3}$. The model's single parameter is thus the spheres volume fraction $f$ whereas the microstructure's characteristic length is $a$.

3.2. Two and three-scale embedded Boolean models. Multiscale models are generated in two steps, using embedded Boolean random sets as described below. These models are examples of Cox Boolean models, where the homogeneous Poisson point process is replaced by a Cox point process (Poisson process with a random intensity $\Theta(x)$ ) (14). In the first step, a microstructure made of a Boolean set of spheres of radius $a^{(L)}$ (called "large spheres") and desired volume fraction of the spheres $f^{(L)}$ is generated. In the second step, a Boolean random set of spheres of radius $a<a^{(L)}$ (called "small" spheres) is generated, under the constraint that all small sphere centers lie inside a large sphere. More precisely, in the second step, sphere centers that are not contained in a large sphere are discarded in the Boolean random process. Additionally, the intensity of small spheres $\theta^{\prime}$ is adjusted as follows, where $f^{(L)}$ is given by equation 3.1 :

$$
f \sim f^{(L)}\left[1-\mathbf{e}^{-\theta^{\prime} \frac{4 \pi}{3} a^{3}}\right] .
$$

A Poisson point process with intensity $\theta$ is used for estimating $\theta^{\prime}$. The inclusions in the final microstructure is the set of small spheres, whereas the rest of the domain is the matrix phase. An example of such embedded Boolean sets process is illustrated in Fig. 10.1 on a planar section of the 3D microstructure. As shown in (19), the 
percolation threshold of a two-scale medium is approximately the product of the two percolation thresholds, and is therefore lowered as compared to the one-scale media . It results into an expected reinforcement of the material in the case of rigid inclusions, as illustrated in this paper.

The volume fraction of the small spheres in the set of large spheres is noted $f^{(S)} \sim f / f^{(L)}$. Two models are considered depending on the choice of $f^{(L)}$. In the iterated model, labelled (IB), $f^{(L)}=f^{(S)}=\sqrt{f}$, allowing for any value of $f$ in the range $0-1$; in the non-iterated model, labelled $(\mathrm{MB}), f^{(L)}$ has a finite fixed value, and $f$ lies between 0 and $f^{(L)}$. In the limit $L \gg a^{(L)}$, both models are parametrized with $f$, and their characteristic length scales are $a^{(L)}$ and $a$. Finally, the percolation threshold for the spheres is noted $f_{p}^{(.)}$for the various models, with $f_{p}^{(B)} \approx 0.2896$ $(40 ; 18), f_{p}^{(I B)}=\left(f_{p}^{(B)}\right)^{2} \approx 0.083$ and $f_{p}^{(M B)}=0.2896 f^{(L)}$ when $f^{(L)} \leq f^{(B)}$. Non-iterated models with specific values of $f^{(L)}$ are noted as $\left(M B-f^{(L)}\right)$, e.g. (MB20) means $f^{(L)}=0.2=20 \%$. Finally, a 3-scales iterated Boolean model (B3), is introduced using the same methodology as for model (IB). Use is made of three embedded Boolean models (L), (S), (S') with decreasing spheres radius and equal volume fractions $f^{(L)}=f^{(S)}=f^{\left(S^{\prime}\right)}=f^{1 / 3}$. Spheres at the length scale (S) are selected when their centers lie inside a sphere at the upper length scale $(\mathrm{L})$, as for model (IB). In turn, spheres at the smallest length scale (S') are kept when their centers lie inside spheres at the length scale (S). The resulting microstructures, made of spheres at the smallest length scale (S'), have a volume fraction $f$. Unlike model (IB), full-field numerical computations for the 3-scales model (B3) were not performed, due to large volumes and discretization involved; only the effective properties of model (B3) are numerically computed, under the assumption of separation of length scales between (L), (S) and ( $\left.\mathrm{S}^{\prime}\right)$, as precised in Sec. 7.1.4.

Consistently with the periodic boundary conditions considered in numerical computations, all Boolean random sets are made periodic. The domain $\Omega$ is seen as the elementary cell of a periodic material, i.e. all coordinate points in the medium are computed modulo $L$. All the results of simulations are provided with the average of the true volume fractions, measured on simulations, instead of using the approximation 3.2 .

Such models, and their 3-scale version were used to model in practical situations the distribution of carbon black nanoparticles in various matrices $(41 ; 5 ; 10)$. In the corresponding references, the problem of estimating the model parameters from image analysis, is addressed by measuring various probabilistic properties like the covariance of binary images.

4. Analytical bounds. The effective properties can be bounded from a limited

statistical information $(2 ; 24)$. The more common bounds are Hashin and Shtrikman's (H-S) $(8 ; 9)$, based on the volume fractions of components in the case of statistically isotropic media. However, many different morphologies can be imagined for given volume fractions. Narrower bounds are obtained by combining a variational principle and trial fields. In practice, it is difficult to obtain useful results beyond the third order. In this work, we make use of Beran's third-order bounds for Boolean media (1).

4.1. Morphological functions to calculate third order bounds. In the case of random materials with elastic isotropic components, and with an isotropic geometry, the third order bounds were given by Beran and Molyneux for the bulk 
modulus $\kappa(1)$ and by McCoy for the shear modulus $\mu$ (31). These bounds are valid for multiphase media, and more generally for elastic moduli modelled by random functions $(2 ; 26 ; 27 ; 24 ; 11)$. In $(15)$ are given examples of bounds of the dielectric permittivity for some random functions.

The third order bounds depend on the moduli of the two phases, the volume fraction $f$, and on two morphological functions introduced by Milton $(33) \zeta_{1}(f)$ and $\eta_{1}(f)\left(0 \leq \zeta_{1}(f) \leq 1\right.$ and $\left.0 \leq \eta_{1}(f) \leq 1\right)$, calculated from the three-point probabilities. We note $P\left(\mathbf{h}_{1}, \mathbf{h}_{2}\right)$ the probability for the three points $\{\mathbf{x}\},\left\{\mathbf{x}+\mathbf{h}_{1}\right\},\left\{\mathbf{x}+\mathbf{h}_{2}\right\}$ to belong to the random set $A$.

The third order bounds of $\kappa$ depend only on the function $\zeta_{1}(f)$. If $\zeta_{1}(f)=1$ or $0, \kappa_{+}=\kappa_{-}\left(=\kappa^{H S-}\left(\zeta_{1}(f)=1\right)\right.$ or $\left.\kappa^{H S+}\left(\zeta_{1}(f)=0\right)\right)$. The Hashin and Shtrikman bounds are therefore a limiting case of third order bounds of $\kappa$. We have the same situations concerning third order bounds of the electrical conductivity.

It turns out that when $\eta_{1}(f)=\zeta_{1}(f)=1(0)$ and when $\kappa_{1}=\kappa_{2}=\infty, \mu_{+}=\mu_{-}=$ $\mu^{H S+}\left(\mu^{H S-}\right)$.

The morphological functions $\zeta_{1}(f)$ and $\eta_{1}(f)$ can be calculated analytically in some cases, but most often numerically. For instance, for a Boolean model of spheres (with a single radius $a$ ), we approximately have:

$$
\begin{gathered}
\zeta_{1}(f) \simeq 0.5615 f(3 ; 42 ; 43 ; 44) \\
\eta_{1}(f) \simeq 0.711 f(3 ; 42 ; 43 ; 44)
\end{gathered}
$$

4.2. Combination of basic random sets. More complex models can be built from elementary ones $(13 ; 41)$. We consider here multi-scale models. An interesting construction is obtained for the intersection of independent random sets . For $A=$ $A_{1} \cap A_{2}$ with volume fractions $f_{1}$ and $f_{2}$ we have:

$$
P\left(\mathbf{h}_{1}, \mathbf{h}_{2}\right)=P_{1}\left(\mathbf{h}_{1}, \mathbf{h}_{2}\right) P_{2}\left(\mathbf{h}_{1}, \mathbf{h}_{2}\right)
$$

for widely separate scales (in particular for $A_{2}$ with a lower scale), we have approximately, noting $\zeta_{H 1}(f), \zeta_{11}\left(f_{1}\right)$ and $\zeta_{12}\left(f_{2}\right)$ the morphological functions of the two-scale medium $A$, of $A_{1}$ and $A_{2}$, and with similar notations for $\eta_{1}(f)$ :

$$
\begin{aligned}
& f(1-f) \zeta_{H 1}(f) \simeq f_{1} f_{2}\left(1-f_{2}\right) \zeta_{12}\left(f_{2}\right)+f_{2}^{3} f_{1}\left(1-f_{1}\right) \zeta_{11}\left(f_{1}\right) \\
& f(1-f) \eta_{H 1}(f) \simeq f_{1} f_{2}\left(1-f_{2}\right) \eta_{12}\left(f_{2}\right)+f_{2}^{3} f_{1}\left(1-f_{1}\right) \eta_{11}\left(f_{1}\right)
\end{aligned}
$$

These approximations, given in (13), are asymptotically exact for an infinite separation of length scales between the two scales. Their derivation, requiring some algebra, is given in (21). When using a Boolean model of spheres for the two scales, it can be shown that $\zeta_{H 1}(f) \geq \zeta_{11}(f)$ (or $\left.\zeta_{22}(f)\right)(13)$, and the third order upper bounds of $\kappa$ and of the conductivity increase when increasing the number of scales. This can be explained by a lowering of the percolation threshold.

If the two structures are built from the same random set with $f_{1}=f_{2}$, we obtain after $n$ iterations of the process involving intersections:

$$
\begin{aligned}
\zeta_{H 1}^{(n)}(f) & =\frac{1+f}{1+f^{1 / n}} \zeta_{1}\left(f^{1 / n}\right) \\
\eta_{H 1}^{(n)}(f) & =\frac{1+f}{1+f^{1 / n}} \eta_{1}\left(f^{1 / n}\right)
\end{aligned}
$$


for $n \rightarrow \infty$ we get asymptotically $\zeta_{H_{1}}^{(n)}(f) \rightarrow \frac{1+f}{2} \zeta_{1}(1)$ and $\eta_{H 1}^{(n)}(f) \rightarrow \frac{1+f}{2} \eta_{1}(1)$. As shown in (16), starting from the complementary set of a Boolean variety of dilated Poisson planes ((12), we have $\zeta_{1}(1)=\eta_{1}(1)=0$ and therefore $\zeta_{H_{1}}^{(\infty)}(f)=\eta_{H 1}^{(n)}(f)=0$. Its complementary set is a union of dilated planes with separate scales, for which $\zeta_{H_{1}}^{(\infty)}(f)=\eta_{H 1}^{(n)}(f)=1$. The obtained limit structure admits two equal third order bounds for the bulk modulus $\kappa$, namely the effective modulus (it is the same result for the conductivity). If $\kappa_{2}>\kappa_{1}$ is attributed to the union of dilated planes,

$$
\kappa^{e f f}=\kappa_{-}=\kappa_{+}=\kappa^{H S+}
$$

If $\kappa_{2}>\kappa_{1}$ is attributed to the complementary set of dilated planes,

$$
\kappa^{e f f}=\kappa_{-}=\kappa_{+}=\kappa^{H S-}
$$

We obtain the construction of an optimal structure corresponding to these bounds, based on the Boolean model. This is an alternative microstructure to the well known Hashin coated spheres. On the other hand, by this process are obtained two separate bounds for the shear modulus $\mu$. However they are very close if the materials are well ordered, that is if $\left(\kappa_{2}-\kappa_{1}\right)\left(\mu_{2}-\mu_{1}\right)>0$.

For an infinite contrast, the upper bound increases to infinity whatever the microstructure is (it becomes irrelevant) when one of the phase becomes rigid. However the evolution of bounds with the microstructures provides useful guidelines to increase the effective properties from multiscale models, as illustrated in the remaining parts of this paper from numerical estimations on simulations.

\section{Local and effective behavior.}

5.1. Mechanical properties. The constitutive behavior of the matrix phase follows an isotropic, compressible linear elastic law, such that the stress and strain tensors are related by the elastic tensor $\mathbb{L}$ :

$$
\sigma(\mathbf{x})=\mathbb{L}: \varepsilon(\mathbf{x}), \quad L_{i j k l}=\kappa \delta_{i j} \delta_{k l}+\mu\left(\delta_{i k} \delta_{j l}+\delta_{i l} \delta_{j k}\right)
$$

where $\sigma$ and $\varepsilon$ are the local stress and strain tensors, respectively, and where $\kappa=1$ and $\mu=1$ are the bulk and shear moduli in the matrix, respectively. The above equation is rewritten in terms of the mean and deviatoric strain and stress components:

$$
\sigma_{m}=3 \kappa \varepsilon_{m}, \quad \sigma^{\prime}=2 \mu \varepsilon^{\prime}, \quad \varepsilon_{i j}^{\prime}=\varepsilon_{i j}-\varepsilon_{m} \delta_{i j}, \quad \sigma_{i j}^{\prime}=\sigma_{i j}-\sigma_{m} \delta_{i j},
$$

where the scalars $\varepsilon_{m}=\varepsilon_{i i} / 3$ and $\sigma_{m}=\sigma_{i i} / 3$ are the mean strain and mean stress components, respectively, whereas we note $\varepsilon^{\prime}$ and $\sigma^{\prime}$ the strain and stress deviatoric parts. This study is concerned with homogenization of strongly contrasted phases, i.e. either porous or rigid inclusions are considered. In the porous phase, the constitutive law reads $\sigma \equiv 0$ with strain undefined, whereas rigid behavior is simulated using the constitutive law $\sigma=h \mathbb{L}: \varepsilon$ where $h=10^{4}$. Such finite value allows one to study the macroscopic behavior of rigidly-reinforced composites for values of the volume fraction of spheres $f$ larger than the percolation threshold $f_{p}$.

The strain derives from a displacement field $\mathbf{u}$ (referred to as the "strain compatibility" condition). Assuming small deformation, this reads:

$$
\varepsilon_{i j}=\frac{1}{2}\left(\partial_{i} u_{j}+\partial_{j} u_{i}\right)
$$


Symmetrically, the stress field satisfies an "equilibrium equation", which reads, in the absence of external forces:

$$
\partial_{i} \sigma_{i j}=0 .
$$

The effective properties are computed by taking averages over the elementary cell $\Omega$. It is assumed that isotropy is recovered at the macroscopic scale:

$$
\begin{aligned}
\varepsilon_{0} & =\langle\varepsilon\rangle, \quad \sigma_{0}=\langle\sigma\rangle=\mathbb{L}^{(0)}: \varepsilon_{0}, \\
\mathbb{L}^{(0)}{ }_{i j k l} & =\kappa_{0} \delta_{i j} \delta_{k l}+\mu_{0}\left(\delta_{i k} \delta_{j l}+\delta_{i l} \delta_{j k}\right)
\end{aligned}
$$

where $\varepsilon_{0}$ and $\sigma_{0}$ are the macroscopic strain and stress, respectively, and $\mathbb{L}^{(0)}$ is the macroscopic elastic tensor. The notations $\kappa_{0}$ and $\mu_{0}$ refer to the effective bulk and shear moduli, respectively, and are normalized by $\kappa$ and $\mu$ in graphs. Alternatively, the more precise notation $\mathbb{L}^{(M)}\left(f, h \mathbb{L}^{(1)}\right)$ (or simply $\mathbb{L}^{(M)}(f, h)$ ) is used for a particular microstructure model $M(\mathrm{M}=\mathrm{B}, \mathrm{IB}, \mathrm{MB})$ with contrast $h$ and sphere volume fraction $f$ (likewise for $\kappa^{(M)}$ or $\left.\mu^{(M)}\right)$. For instance, it is obvious that:

$$
\mathbb{L}^{(I B)}(f, h)=\mathbb{L}^{(B)}\left(\sqrt{f}, \mathbb{L}^{(B)}(\sqrt{f}, h)\right) .
$$

To estimate numerically $\mathbb{L}^{(M)}$, both hydrostatic and shear strain loading are considered. More precisely, the macroscopic strain takes one of the two forms

$$
\left(\varepsilon_{0}\right)_{i j}=\overline{\varepsilon_{0}} \delta_{i j}, \quad\left(\varepsilon_{0}\right)_{i j}=\overline{\varepsilon_{0}}\left(1-\delta_{i j}\right),
$$

where $\overline{\varepsilon_{0}}=1$ is a scalar.

5.2. Conducting properties. For the sake of comparison, it is interesting to consider the mechanical as well as the conducting properties of heterogeneous multi-scale materials made of infinitely-contrasted phases, i.e. containing perfectlyinsulating or highly-conducting inclusions. Linear conductivity is assumed for the material local response. The electric and current vector fields are noted $E$ and $J$, respectively, such that, in the matrix:

$$
J(\mathbf{x})=\chi E(\mathbf{x}),
$$

where $\chi=1$ is the electric conductivity. In insulating inclusions, the local current is zero $(J=0)$ whereas the electric field $E$ is undefined; in highly-conducting inclusions, the local response is defined as $J=$ response is defined analogously to the elastic case. The electric field derives from a potential $\Phi$, whereas the current is divergence-free:

$$
E_{i}=-\partial_{i} \Phi, \quad \partial_{i} J_{i}=0 .
$$

Assuming an isotropic microstructure, the corresponding macroscopic properties are defined as:

$$
J_{0}=\langle J\rangle=\chi_{0} E_{0}, \quad E_{0}=\langle E\rangle,
$$

where $J_{0}$ and $E_{0}$ are the macroscopic current and electric fields and $\chi_{0}$ is the effective conductivity. The latter is computed by applying a difference of potential along an arbitrary direction $e_{x}$ :

$$
E_{0}=\overline{E_{0}} e_{x},
$$

where $\overline{E_{0}}$ is a scalar. 
6. FFT numerical estimates. The numerical approach used in this work is based on the FFT method introduced in (37) ("direct scheme"), and specifically on the "augmented Lagrangian" algorithm proposed in (32). The latter offers better convergence in the case of strongly-contrasted properties than the original direct scheme. The microstructure is simulated by a cube made of $L \times L \times L$ voxels, and digitized with resolutions $L=512$ or $L=750$. To each voxel is assigned a phase (either inclusion or matrix), with its appropriate local elastic moduli or conductivity. The FFT method consists of a fixed-point algorithm based on the Lippmann-Shwinger equations. For linear elastic problems, the latter reads:

$$
\begin{aligned}
\varepsilon(x) & =\varepsilon_{0}+\int \mathrm{d}^{3} x^{\prime} \mathbb{G}^{(0)}\left(x-x^{\prime}\right): \tau(x), \\
\tau(x) & =\left[\mathbb{L}(x)-\mathbb{L}^{(0)}\right]: \varepsilon(x),
\end{aligned}
$$

where $\mathbb{G}^{(0)}$ is Green's strain functions, $\mathbb{L}^{(0)}$ is an homogeneous reference tensor and $\tau$ is the strain polarization field. The convolution in Eq. (6.1a) accounts for the admissibility and stress equilibrium conditions (the strain field derives from a displacement and stress is divergence-free) and is computed in the Fourier domain, whereas the local properties are accounted for in $(6.1 \mathrm{~b})$ and are computed in the real space. The error criterion

$$
\eta=\max _{\mathbf{q}}\left\{\frac{\left|\mathbf{q}_{i} \cdot \sigma_{i j}(\mathbf{q})\right|}{\sigma_{0}}\right\},
$$

where $\mathbf{q}$ are Fourier frequencies, is used to monitor convergence at each iteration. Iterations are typically stopped when $\eta \approx 10^{-5}$. It has been observed that the convergence properties strongly depend on the choice of the reference tensor $\mathbb{L}^{(0)}$. The latter is chosen in the form $\mathbb{L}^{(0)}=t \mathbb{L}$ where $\mathbb{L}$ is the elastic tensor in the matrix, and $t$ is optimized on microstructures with small resolution (e.g. a grid of $32^{3}$ voxels). It is found that $t$ varies from $10^{-1}$ for porous media to $10^{3}$ for rigidly-reinforced microstructures. The number of iterations varies from 40 to 500 iterations, depending on the microstructure.

For each microstructure, four random microstructure samples were used to perform FFT computations. From the variance over simulations a confidence interval is calculated and given on the plots. Overall, a relative precision of about 1 to $5 \%$ is achieved for nearly all points. In effect, the size of one sample alone is sufficiently high, as compared to the highest characteristic length in the microstructure, to alleviate for what would be a lack of representativity at smaller sizes. The representativity of the samples is discussed in more details in Sec. 9.3.

\section{Mechanical behavior.}

\subsection{Rigidly-reinforced composites.}

7.1.1. Behavior in the dilute limit . In this section, the behavior of various multiscale Boolean models is investigated in the dilute limit $(f \rightarrow 0)$. Emphasis is made on the effective bulk modulus $\kappa_{0}$ of rigidly-reinforced media. In order to derive analytical results, separation of length scales is assumed.

Consider a two-scale microstructure (M) made of two embedded Boolean models with inclusion concentrations $f_{1}$ and $f_{2}$ at the smallest and largest scales, respectively, where the total volume fraction of rigid inclusions is $f=f_{1} f_{2}$. Its effective elastic 
tensor $\mathbb{L}^{(M)}$ is obtained from the formula:

$$
\mathbb{L}^{(M)}(f)=\mathbb{L}^{(B)}\left(f_{2}, \mathbb{L}^{(B)}\left(f_{1},+\infty\right)\right),
$$

where it is recalled that the local behavior of the matrix is defined by its elastic tensor $\mathbb{L}$ of moduli $\kappa=\mu=1$. Accordingly, the iterated Boolean model (IB) corresponds to $f_{1}=f_{2}=\sqrt{f}$, whereas in the non-iterated two-scales models (MB), $f_{2}$ is fixed and $f_{1}=f / f_{2}$. For the Boolean model (B) with general elastic tensor $\mathbb{L}^{(\text {inc })}$ in inclusions, the effective properties $\mathbb{L}^{(B)}\left(f, \mathbb{L}^{(\text {inc })}\right)$ may be rewritten as $\mathbb{L}^{(B)}\left(f, h, h^{\prime}\right)$ in terms of the phase contrasts $h=\kappa^{(\mathbf{i n c})} / \kappa$ and $h^{\prime \text { (inc) }} / \mu$ where $\kappa^{(\mathbf{i n c})}, \mu^{(\mathbf{i n c})}$ are the inclusions bulk and shear moduli, respectively. Consequently, the behavior of the bulk modulus $\kappa^{(B)}$ of $\mathbb{L}^{(B)}$ in the dilute-limit is given by Hashin-Shtrikman's lower bounds (HS) up to first-order correction. Assuming an analytical expansion up to order 2:

$$
\kappa_{0}^{(B)} / \kappa=1+\frac{7(h-1)}{4+3 h} f+b\left(h, h^{\prime}\right)^{2}+O\left(f^{3}\right)
$$

where the factor $b\left(h, h^{\prime 2}\right)$ when $h \rightarrow 1$, as (HS) bounds are exact up to the secondorder in contrast. In the infinite-contrast limit $\left(h=h^{\prime} \rightarrow \infty\right), b\left(h, h^{\prime}\right) \rightarrow \beta$ with $\beta \geq 7 / 3$ according to (HS) lower bounds. For a uniform probability distribution of well-separated spheres (45) gives the approximate value $\beta=287 / 108 \approx 2.66>7 / 3$, whereas an exact formula is given in (4). The expansion in the dilute limit of a twoscales materials $(\mathrm{M})$ depends on the behavior of $f_{1}$ and $f_{2}$ as $f \rightarrow 0$, or equivalently that of $f$ and $f_{1}$, the latter being interpreted as a dispersion parameter. Large values of $f_{1}$ correspond to highly non-uniform distribution of heterogeneities with rigid particles grouped in aggregates, whereas the spatial distribution of heterogeneities is more uniform at low values of $f_{1}$. Assuming a power law behavior $f_{1} \sim p f^{r}$ so that $f_{2} \sim f^{1-r} / p$ with $0 \leq r \leq 1$, use is made of Eqs. (7.2) and (7.1):

$$
\kappa^{(M)} / \kappa=1+(7 / 3) f\left[1+\left(f / f^{*}\right)^{r}\right]+O\left(f^{1+2 r}+f^{2}\right),
$$

where $f^{*}=[p(3 \beta / 7-1)]^{-1 / r}$. The next-to-leading order term in $\sim f^{1+r}$ has a positive prefactor, hence it occurs that, as expected, two-scales media have stronger elastic properties: in the dilute limit $\kappa_{0}^{(M S)}>\kappa_{0}^{(B)}$. Also, the elastic reinforcement is larger when inclusions are non-uniformly distributed, i.e. when $p$ increases or $r$ decreases at constant $f$. In the non-iterated Boolean media (MB) considered in this work, $f_{2}$ is constant, i.e. $r=1$. For this value of $r$, an analytical expansion is recovered for the bulk modulus. Compared to the one-scale Boolean model, a small reinforcing effect is observed, at the second-order in the rigid phase volume fraction $\left(\sim f^{2}\right)$. In the opposite limit $r \rightarrow 0$, or equivalently when the volume fraction $f_{1}$ at the small scale is constant, the terms in $f^{1+r}$ as well as terms embedded in $O\left(f^{1+2 r}\right)$ become linear in $f$. Although the overall linear correction depends on the entire expansion of Eq. (7.2), it may be derived at first-order in $f_{1}$. At fixed $f_{1} \ll 1$ and $f \rightarrow 0$ :

$$
\kappa^{(r=0)} / \kappa=1+\alpha\left(f_{1}\right) f+O\left(f^{2}\right), \quad \alpha\left(f_{1}\right) \approx 7 / 3\left[1+f_{1} / f_{1}^{*}+O\left(f_{1}^{2}\right)\right],
$$

where $f_{1}^{*}=(3 \beta / 7-1)^{-1}$. Accordingly, in a two-scale Boolean media, a highly nonuniform dispersion $(r=0)$ leads to strongly reinforced elastic properties in the dilute limit, in the sense that the first-order correction depends on interaction between neighboring particles, and is different from Eshelby's dilute estimate for a single inclusion.

The dilute regime is now investigated for $n$-scales $(n \geq 2)$ Boolean microstructures, noted (IB-n). In case of $n$-scales, the centers of the smallest spheres (making 
the reinforcement or the pores), are located inside the intersection of the $n-1$ larger scales. Again, we generate a Cox Boolean model where the random intensity $\Theta(x)$ of the Poisson point process is proportional (factor $\theta$ ) to the indicator function of the $n-1$ larger scales. For simplicity, it is assumed that the spheres concentrations of Boolean models is the same at all scales, i.e. $f_{1}=\ldots=f_{n}=f^{1 / n}$, where $f_{i}$ is the sphere concentration at scale $i$. Said otherwise, such a microstructure corresponds to a random hierarchical model where spheres are placed according to Poisson point processes with intensities satisfying, for every scale $i, \theta_{i} a_{i}^{3}=C t e$. The overall behavior is that of the iterated Boolean model (IB) when $n=2$. The dilute regime is expanded as, using recursively Eq. (7.1):

$$
\kappa^{(I B-n)} / \kappa=1+(7 / 3) f\left[1+f^{1 / n} / f_{1}^{*}\right]+O\left(f^{(n+2) / n}\right) .
$$

Accordingly, in the dilute limit, the reinforcement occurs at the next-to-leading order term in $\sim(7 / 3) f^{1 / n} / f_{1}^{*}$. This reinforcement becomes quasi-linear when $n \gg 1$, as for a two-scale microstructure with $f=1$ and $r=1 / n \ll 1$. Therefore, the local concentration at the smallest scale controls the reinforcement in the dilute limit.

7.1.2. Separation of length scales. The effective bulk (resp. shear) modulus of two iterated Boolean models (IB-4) and (IB-10) is plotted on top (resp. bottom) of Fig. 10.2 as a function of the volume fraction $f$ of rigid spheres, and compared with the elastic properties of the one-scale model (B). Models (IB-4) and (IB-10) differ only by their scales ratio $a^{(L)} / a=4,10$, respectively. In order to estimate the lower value of scale ratio at which separation of length scales occur, comparison is made with the effective elastic tensor $\mathbb{L}^{(F C)}$ of a finite-contrast Boolean model $(\mathrm{FC})$ that simulates an iterated Boolean model with infinitely-separated length scales (i.e. $a^{(L)} / a=+\infty$ ). The latter is a one-scale Boolean model (B) with sphere volume fraction $\sqrt{f}$, and where the local properties of the spheres are given by the effective properties of model (B) at the same volume fraction of spheres $\sqrt{f}$. Equivalently, formula (5.7) is implemented numerically as two FFT computations of one-scale media. Accounting for the variable change $f \rightarrow \sqrt{f}, \mathbb{L}^{(F C)}(f) \equiv \mathbb{L}^{(I B)}(f)$.

As expected, the non-uniform distribution of heterogeneities in the iterated twoscales models (IB-4) and (IB-10) results, in the rigid case, in reinforced elastic behavior compared to the one-scale Boolean model (B), at all volume fractions. For the iterated model (IB), significant reinforcement occurs for volume fractions greater than the percolation threshold (i.e. $f \geq f_{p}^{(I B)}=f_{p}^{2} \approx 0.083$ ) and diminishes with $f$ at large volume fractions $(f \geq 0.4)$. For volume fractions up to $f \approx 0.4$, separation of length scales is obtained at a good precision when $a^{(L)} / a=10$. It is noted that for large volume fractions, separation of length scales requires very large resolutions, as the estimates at $a^{(L)} / a=4$ and 10 are almost identical for $f>0.6$. In the remaining part of the paper, the scale ratio in multi-scale models is fixed to $a^{(L)} / a=10$.

A practical consequence from an engineering point of view is that for a large contrast of properties it is possible to homogenize the effective properties by iterations over the scales (starting from the smallest ones, and updating the properties of the matrix phase at each iteration), as soon as their separation reaches an order of magnitude. Therefore a simplification of the numerical homogenization tools by iterations can be proposed in the case of a factor 10 in each intermediate scale. Lower separations of scales should be required for a lower contrast in properties. This scheme was applied in the previous section about dilute concentrations 7.1.1.

7.1.3. Effective behavior. In Fig. (10.3), the effective elastic moduli $\kappa_{0}$ and $\mu_{0}$ of various multiscale Boolean models (IB), (MB-20), (MB-30), (MB-50) are plotted 
as functions of $f$, the volume fraction of the rigid phase. Comparisons are made with the one-scale model (B) as well as the third-order (TOB) and Hashin-Shtrikman (HS) analytical bounds. A log-lin scale is used to emphasize the elastic behavior of the material for the entire range of volume fractions, and the third-order (Beran's) upper bounds correspond to a Boolean microstructure with infinitely many scales. It turns out that for the contrast used in our calculations, HS and TOB analytical bounds are inefficient, being to far apart. Depending on the volume fractions of spheres $f$, the bulk moduli of Boolean models (MB-20), (MB-30) and (MB-50), i.e. models with fixed large-scale parameter $f^{(L)}$, is higher or smaller than that of the iterated Boolean model (IB). For small values of $f$, the former is less than the latter, whereas both coincide at finite volume fraction $f=f^{(1)}=\left(f^{(L)}\right)^{2}=0.04,0.09$ and 0.25 , for models (MB-20), (MB-30) and (MB-50), respectively. Non-iterated models also coincide with the one-scale Boolean model (B) at the largest volume fraction $f=f^{(L)}$. This suggests that there exists a volume fraction $f^{(2)} \neq f^{(1)}$ at which the bulk modulus of model (IB) is equal to that of non-iterated microstructures (MB-20), (MB-30) and (MB$50)$. The curves presented in Fig. (10.3) show that $f^{(2)}>f^{(1)}$ with $f^{(2)} \approx 0.1,0.18$ and 0.35 for microstructures (MB-20), (MB-30) and (MB-50), respectively. Hence, the elastic moduli of non-iterated Boolean models is successively smaller, larger and smaller again than that of the iterated Boolean model for increasing values of the rigid fraction $f$.

7.1.4. Two and three-scale Boolean microstructures. Estimates of the effective bulk and shear moduli $\kappa_{0}$ and $\mu_{0}$ of one, two and three-scale iterated Boolean models (B), (IB) and (B3), respectively, are shown in Fig. (10.4), where a lin-log scale is used. The mechanical behavior of the three-scales model (B3) is computed numerically using a one-scale Boolean model with finite-contrast and applying Eq. 5.7. These results are compared with Hashin-Shtrikman's (HS) and Beran's thirdorder upper and lower bounds (TOB, TOB $\left.{ }^{\infty}\right)$. Two of Beran's bounds are represented. The lowest series of bounds corresponds to a one-scale Boolean microstructure (TOB), whereas the highest series of bounds are that of a Boolean model with infinitely many separated scales $\left(\mathrm{TOB}^{\infty}\right)$. As such, it is noted that any "iterated" microstructure made of embedded Boolean models have elastic properties that are strictly lower than Hashin-Shtrikman's estimates. At large sphere volume fractions $f \geq 0.6$, the three-scales model (B3) has bulk and shear moduli close to that of Beran's bounds, however, as was observed in Fig. (10.2), separation of lengths scales requires very high scale ratios in that range of volume fractions. In any case, it is noted that the reinforcement effect between models (IB) and (B3) is very high at all volume fractions and in particular higher than the one occurring between models (IB) and (B). For instance, when $f \approx 30 \%$, the reinforcement ratio for the bulk modulus is about 8 between models (B3) and (IB), and about 3 between (IB) and the one-scale model (B).

Field maps. Field maps of the strain fields as computed by FFT are shown in Fig. 10.8 and 10.9 for the one-scale and two-scales materials (B) and (IB), respectively on the left and right columns, when the microstructure is subjected to either hydrostatic or shear loadings. The stress component parallel to the applied loading (that is, $\varepsilon_{m}$ and $\varepsilon_{x y}$ for pressure and shear, respectively), is shown, at increasing volume fractions $f=0.2,0.3$ and 0.6 (top to bottom respectively). To make the fields patterns conspicuous in the maps, each strain component is thresholded. More exactly, referring to the strain field distribution in the matrix, the colormaps used here correspond to windows containing $90 \%$ of the fields values and centered around the 
most frequently occurring strain field value. It is emphasized that such maps are not necessarily indicative of the materials effective behavior, as high values of the strain fields contribute significantly to the average strain.

It is noted that the strain field is close to 0 in large regions located in-between quasi-rigid spheres that are close to one another (sphere aggregates). Such "diffusive" effect takes place at volume fraction $f=0.2$ (right-top map) and is slightly more important at volume fraction $f=0.3$ (right middle map).

7.2. Porous media. Numerical FFT results for porous mutiscale composites, as well as Hashin-Shtrikman's (HS) and Beran's (TOB) upper bounds, are shown in Fig. (10.5). The effective behavior, i.e. the macroscopic bulk and shear moduli $\kappa_{0}$ and $\mu_{0}$, respectively, are plotted as a function of the porosity, or sphere volume fractions, $f$, for the entire range of sphere concentrations allowed by the various models: onescale model (B), iterated Boolean model (IB) and three non-iterated two scale models (MB20), (MB30) and (MB50). The third-order bound (TOB) is an exact upperbound for the one-scale Boolean model (B). It gives good estimates of the elastic behavior of the latter at porosities up to about $f \approx 0.2$. At large porosities, both Hashin-Shtrikman's and Beran's bounds are qualitatively different from FFT data. They percolate at $f=1$ with a finite slope, i.e. with the effective elastic moduli are proportional to $1-f$. According to FFT computations, Boolean models (B) and (IB) percolate at volume fractions strictly less than 1 with a zero slope, i.e. the effective elastic moduli are proportional to a power of $f_{p}^{(B, I B)}-f$ higher than 1 . Close to the percolation threshold, deformation is facilitated by large clusters of voids and stress concentrates in a small subset of the material (Willot and Jeulin, 2009). This phenomenon is even stronger for the iterated model (IB) than it is for the one-scale model, and correspond to higher levels of field heterogeneities (see bottom maps in Fig. 10.10).

The elastic properties of non-iterated models (MB-20), (MB-30) and (MB-50) are close to that of the iterated model (IB) and significantly lower than that of the one-scale model (B). A similar weakening is observed for the bulk and shear moduli. From a practical point of view, it means that the counterpart of a reinforcement of a material by a multiscale distribution of rigid inclusions would result into a larger degradation of the elastic moduli by damage (from the break-up of the interface or of inclusions), in comparison to the one scale situation. Contrary to composites made of rigid spheres, a non-uniform distribution of voids weakens the material's elastic properties: the elastic moduli of two-scales media are smaller than that of the one-scale model, at all volume fractions. Accordingly, in a two-phases composite made of inclusions embedded in a continuous matrix, the role of the former on the overall elastic properties is amplified as their spatial distribution becomes non uniform. In particular, the percolation threshold diminishes when the number of separated scales in Boolean models increases. The mechanical percolation threshold for rigidlyreinforced media is about $f \approx 0.29$, which corresponds to the spheres geometrical percolation. For porous media, however, it corresponds to the matrix percolation and is close to 1 (about $f \approx 0.97(6 ; 18)$ ), so that the percolation threshold for model (IB) is about 0.95 . Hence, at moderated volume fractions, the weakening effect of model (IB) compared to model (B) is small (less than $25 \%$ for $f<0.5$ ). A strong weakening of the material elastic properties is observed at large volume fractions only, close to the percolation threshold of the solid phase of model (IB). By comparison, an increase of the elastic properties of $150 \%$ is observed between models (B) and (IB) for rigidly-reinforced media at volume fractions $f=0.2$. Such reinforcement depends 
on the contrast chosen here $\left(10^{4}\right)$ and should increase as the spheres become ideally rigid.

Field maps of the stress fields as computed by FFT are shown in Fig. 10.10 and 10.11 for the one-scale and two-scales materials (B) and (IB), respectively on the left and right columns, when the microstructure is subjected to either hydrostatic or shear loadings. The stress component parallel to the applied loading (that is, $\sigma_{m}$ and $\sigma_{x y}$ for pressure and shear, respectively), is shown, at increasing volume fractions $f=0.2$, 0.3 and 0.6 (top to bottom respectively). Fields are thresholded in a similar way as for the strain components to rigly-reinforced media (see Sec. 7.1.4).

It is noted that, for the iterated Boolean model (IB) at porosity $f=0.2$, the mean stress field is small in regions of the matrix located in-between pores, whereas higher values are located both inside and in-between aggregates of voids (top-right map of Fig. 10.10). Accordingly, regions of the matrix located inside clusters of voids and not well connected to other matrix regions are less solicited, or equivalently act as a third phase with lower elastic moduli.

8. Electrical behavior. The effective conductivity $\chi_{0}$ of multiscale Boolean models (IB), (MB-20), (MB-30), (MB-50) and of the one-scale Boolean model (B) are plotted as functions of the volume fraction of highly-conducting (resp. perfectlyinsulating) spheres at the top (resp. bottom) of Fig. 10.6. Exact upper-bound are provided for comparison: Hashin-Shtrikman's upper and lower bounds and, for highlyconducting spheres, Beran's third-order upper-bound for an iterated model with an infinite number of scales (TOB). An estimate of the response of the infinitely iterated model $\mathrm{IB}^{\mathrm{inf}}$, detailed below, is provided as well. As compared to the elastic properties, qualitatively similar behavior is found. When spheres are highly conducting, the non-uniform dispersion of inclusions, as in models IB or MB, leads to higher overall conductivity, whereas, when inclusions are perfectly-insulating, the macroscopic conductivity is lower in multiscale microstructures than for the one-scale Boolean model (B). Such effect is however less important in the latter case for volume fractions up to $f=0.7$, as there is no electrical percolation threshold in that range when the heterogeneities are insulating. Finally, compared to the mechanical case, similar conclusions are drawn for the behavior of the non-iterated Boolean models (MB-20), (MB-30) and (MB-50) as compared to the iterated model (IB), when the spheres are highly conducting. At very small sphere volume fractions and around $f=f^{(L)}$, their effective properties are smaller than that of the iterated model (IB), whereas they have lower effective properties than model (IB) in-between.

In order to compare quantitatively the electrical responses of the one-scale and multi-scale Boolean models, FFT results are now used to derive scaling laws near the percolation threshold. For the effective conductivity $\chi_{0}^{(B)}$ of the one-scale Boolean model (B), the following behavior is observed for sphere volume fractions close to $f \approx f_{p}^{(B)}$, the spheres percolation threshold in model $(\mathrm{B})$ :

$$
\begin{aligned}
\chi_{0}^{(B)} /(h \chi) & \sim 1.5\left[f-f_{p}^{(B)}\right]^{1.5}, \quad f \gtrsim f_{p}^{(B)}, \\
\chi_{0}^{(B)} / \chi & \sim 0.1\left[f-f_{p}^{(B)}\right]^{-1.65}, \quad f \lesssim f_{p}^{(B)},
\end{aligned}
$$

where $h \gg 1$ is the phase contrast and $\chi$ the local conductivity in the matrix. Note that the macroscopic conductivity $\chi_{0}^{(B)}$ is normalized with the highest local conductivity among percolating phases, i.e. $\chi$ for the matrix or $h \chi$ for spheres, when $f<f_{p}^{(B)}$ 
and $f>f_{p}^{(B)}$, resp. The exponent 1.5 found in upper-limit $f>f_{p}^{(B)}$ is close to many experimental results: scaling laws of exponents 1.5 and 1.4 were obtained for two polyaniline inorganic salt composites (39), whereas earlier experiments carried out in (38) with a packed set of conducting and non-conducting spheres lead to an exponent close to 1.7. For the iterated two-scales Boolean model (IB), it is found from the results of numerical simulations that:

$$
\begin{aligned}
\chi_{0}^{(I B)} /(h \chi) & \sim 3.0\left[f-f_{p}^{(I B)}\right]^{3.0}, \quad f \gtrsim f_{p}^{(I B)}, \\
\chi_{0}^{(I B)} / \chi & \sim 0.44\left[f-f_{p}^{(I B)}\right]^{-0.35}, \quad f \lesssim f_{p}^{(I B)},
\end{aligned}
$$

where $f_{p}^{(I B)}=\left(f_{p}^{(B)}\right)^{2} \approx 0.08$ is the percolation threshold of the iterated two-scales model. It is noted that the observed exponents are very different for the one-scale and two-scales models (IB) and (B). As in Sec. 7.1.1, appropriate analytical expansions are used to interpret this result. The macroscopic conductivity of model (B) is expanded first in the highly-conducting limit $h \rightarrow \infty$, then in the limit $f \rightarrow f_{p}^{(B)}$ as:

$$
\frac{\chi_{0}^{(B)}(f, h)}{h \chi}=\left[\alpha\left(f-f_{p}^{(B)}\right)^{\gamma}+o\left(f-f_{p}^{(B)}\right)^{\gamma}\right]+O(1 / h), \quad f \gtrsim f_{p}^{(B)}
$$

where $\alpha, \gamma$ are the prefactor and exponent of the scaling law for $f>f_{p}^{(B)}$ and where the term in $O(1 / h)$ depends on $f$. Assuming separation of length scales, the scaling law for model (IB) is given by $\chi_{0}^{(I B)}(f)=\chi_{0}^{(B)}\left(\sqrt{f}, \chi_{0}^{(B)}(\sqrt{f}, h)\right)$ such that in the limit $h \rightarrow \infty$ :

$$
\frac{\chi_{0}^{(I B)}(f, h)}{h \chi} \sim \frac{\alpha^{2}}{\left(2 f_{p}^{(B)}\right)^{2 \gamma}}\left[f-f_{p}^{(I B)}\right]^{2 \gamma}, \quad f \gtrsim f_{p}^{(I B)} .
$$

Accordingly, the exponent of the scaling laws for the iterated two-scales Boolean model is 2 times greater than for the one-scale model, consistently with the numerical fit (8.3). The analytical prefactor given above differs from the factor estimated numerically (3.0 vs. 11.6, resp.), however the precision of the FFT data must be taken with care, as the percolation threshold for digitized microstructures (dependant on the grid size) is different from the percolation threshold in a continuum model, and $h$ is not strictly infinite in FFT computations. Using Eq. (8.5) recursively, Eq. (8.6) is generalized as:

$$
\frac{\chi_{0}^{(I B-n)}(f, h)}{h \chi} \sim \frac{\alpha^{n}}{n^{n \gamma}\left(f_{p}^{(B)}\right)^{n(n-1) \gamma}}\left[f-\left(f_{p}^{(B)}\right)^{n}\right]^{n \gamma}, \quad f \gtrsim\left(f_{p}^{(B)}\right)^{n},
$$

for the $n$-scales iterated model (IB-n). Accordingly, an increase of the number of scales leads to stiffer behavior in the upper percolation limit. The domain of validity of such expansion however rapidly shrinks to 0 when $n \rightarrow \infty$. Indeed, the response for the material with infinitely many scales is governed by the behaviour near $f=1$ of the one-scale model. Assume that, in the matrix dilute limit $f \rightarrow 1$ and at $h \rightarrow \infty$ :

$$
\frac{\chi_{0}^{(B)}(f, h)}{h \chi}=1-p(1-f)+o(1-f)^{2}+O(1 / h), \quad f \rightarrow 1,
$$


where, according to Beran's third-order bound $p=(1 / h \chi)\left[\partial \chi_{0}^{(B)} / \partial f\right]_{f=1} \approx 1.88211$. Then, for the multi-scale model (IB- $n$ ):

$$
\frac{\chi_{0}^{(I B-n)}(f)}{h \chi}=\left[1-p\left(1-f^{1 / n}\right)+o\left(1-f^{1 / n}\right)^{2}\right]^{n}, \quad f \rightarrow 1,
$$

where, due to the $f^{1 / n}$ term, the domain of validity of such expansion increases with $n$. As such, when $n=\infty$ and $h=\infty$, the following estimate is found:

$$
\frac{\chi_{0}^{(I B-\infty)}(f)}{h \chi}=f^{p} .
$$

The estimate above is plotted as a function of the sphere volume fractions in Fig. 10.6 (curve marked $\mathrm{IB}^{\mathrm{inf}}$ ). Since 8.8 is only valid when $h=\infty$, it is modified as $h f^{p}$ to take into account the finite value of $h$. It was verified that this estimate is less than the upper third-order bound of the infinite intersection of Boolean models of spheres (TOB).

As seen in Figs. 10.2, 10.3 and 10.4, the effective conductivity is much more sensitive to percolation effects than the elastic properties. Close to the percolation threshold, the following scaling laws are observed for the effective elastic moduli of the one-scale Boolean model (B):

$$
\begin{aligned}
\frac{\kappa_{0}}{h \kappa} & \sim 2.4\left[f-f_{p}^{(B)}\right]^{2.3}, \quad f \gtrsim f_{p}^{(B)}, \\
\frac{\kappa_{0}}{\kappa} & \sim 0.32\left[f-f_{p}^{(B)}\right]^{-1.0}, \quad f \lesssim f_{p}^{(B)}, \\
\frac{\mu_{0}}{h \mu} & \sim 2.4\left[f-f_{p}^{(B)}\right]^{2.5}, \quad f \gtrsim f_{p}^{(B)}, \\
\frac{\mu_{0}}{\mu} & \sim 0.31\left[f-f_{p}^{(B)}\right]^{-1.1}, \quad f \lesssim f_{p}^{(B)} .
\end{aligned}
$$

As was observed in (46), the scaling law for $f>f_{p}^{(B)}$ is valid for a large range of volume fractions.

According to the remark above, the exponent in the scaling law is smaller (resp. greater) when $f>f_{p}^{(B)}$ (resp. $f<f_{p}^{(B)}$ ) for the electric behavior than for the elastic moduli. As for multi-scale materials, the interpretation of the FFT data was difficult due to the high grid precision required to estimate such scaling laws. Such questions are saved for future studies.

9. Statistical fluctuations and RVE. The maps of the fields obtained by iterations of FFT can be used for some detailed statistical study that will be reported elsewhere. The probability distributions of stresses, strains (elasticity), or electric current and field can be accessed from the simulations. From these distributions, the statistical variances of the fields are available and are an intrinsic measure of their variability. Furthermore, these second order statistics provide us with information on the representativity of the simulated microstructures, according to the corresponding fields. In this section the representativity of the effective bulk and shear modulus (resp. electric conductivity) estimated on a bounded domain of a microstructure is addressed. To compute the so-called "Representative Volume Element" (22; 17) (RVE) we make use of a geostatistical approach based on the experimental determination of the integral range (28) from numerical simulations. 
9.1. The integral range . We consider fluctuations of average values over different realizations of a random medium inside the domain $B$ with the volume $V$. In geostatistics, it is well known that for an ergodic stationary random function $Z(x)$, with mathematical expectation $E(Z)$, one can compute the variance $D_{Z}^{2}(V)$ of its average value $\bar{Z}(V)$ over the volume $V$ as a function of the central covariance function $\bar{C}(h)$ of $Z(x)(28)$ by :

$$
D_{Z}^{2}(V)=\frac{1}{V^{2}} \int_{V} \int_{V} \bar{C}(x-y) d x d y
$$

where

$$
\bar{C}(h)=E\{(Z(x)-E(Z))(Z(x+h)-E(Z))\}
$$

For a large specimen (with $V \gg A_{3}$ ), equation (9.1) can be expressed to the first order in $1 / V$ as a function of the integral range in the space $R^{3}, A_{3}$, by

$$
\begin{aligned}
D_{Z}^{2}(V) & =D_{Z}^{2} \frac{A_{3}}{V} \\
\text { with } A_{3} & =\frac{1}{D_{Z}^{2}} \int_{R^{3}} \bar{C}(h) d h
\end{aligned}
$$

where $D_{Z}^{2}$ is the point variance of $Z(x)$ (here estimated on simulations) and $A_{3}$ is the integral range of the random function $Z(x)$, defined when the integral in equations (9.1) and (9.3) is finite. The asymptotic scaling law (9.2) is valid for an additive variable $Z$ over the region of interest $V$.

To estimate the effective elasticity or conductivity tensors from simulations, we have to compute the averages $\langle\sigma\rangle$ and $\langle\varepsilon\rangle$ (elastic case) or $\langle J\rangle$ and $\langle E\rangle$. For the applied boundary conditions the modulus is obtained from the estimations of a scalar, namely the mean stress, strain, current, or electric field. Therefore the variance of the effective property follows the equation (9.2) when the integral range $A_{3}$ of the relevant field is known.

Since the theoretical covariance of the fields $(\sigma$ or $\varepsilon$ ) is not available, the integral range can be estimated according to the procedure proposed by Georges Matheron for any random function (30): working with realizations of $Z(x)$ on domains $B$ with an increasing volume $V$ (or in the present case considering subdomains of large simulations, with a wide range of sizes), we can estimate the parameter $A_{3}$ by fitting the obtained variance according to the expression (9.2). The point variance $D_{Z}^{2}$ of the corresponding field is directly estimated from the experimental variance of the field.

Concerning the statistical precision of the effective properties, the crucial parameter is the variance $D_{Z}^{2}(V)$, more than the point variance $D_{Z}^{2}$. Even starting from a single large realization, the integral range $A_{3}$ can be estimated from the variance of subvolumes at different scales. The variance of the average decreases with the scale $V$, as given by Eq. 9.3, valid even for a single realization by some ergodicity effect. To estimate the effective properties with a given precision, as detailed in Sec. 9.2, we can alternatively use some large size simulations, or many small size simulations, provided no bias is introduced by the boundary conditions.

9.2. Practical determination of the size of the RVE. When considering a material as a realization of a random set or of a random function RF, the idea that there exists one single possible minimal RVE size must be left out. Instead, the size of a RVE can be defined for a physical property $Z$, a contrast and, above all, a 
given precision in the estimation of the effective properties depending on the number of realizations that one is ready to generate. By means of a standard statistical approach, the absolute error $\epsilon_{a b s}$ and the relative error $\epsilon_{\text {rela }}$ on the mean value obtained with $n$ independent realizations of volume $V$ are deduced from the interval of confidence by:

$$
\epsilon_{a b s}=\frac{2 D_{Z}(V)}{\sqrt{n}} ; \epsilon_{\text {rela }}=\frac{\epsilon_{a b s}}{Z}=\frac{2 D_{Z}(V)}{Z \sqrt{n}},
$$

where the factor 2 results from the standard $95 \%$ confidence interval for normally distributed data. The size of the RVE can now be defined as the volume for which for instance $n=1$ realization is necessary to estimate the mean property $Z$ with a relative error $\epsilon_{\text {rela }}=1 \%$, provided we know the function $D_{Z}(V)$. Alternatively, we can decide to operate on smaller volumes (provided no bias is introduced by the boundary conditions), and consider $n$ realizations to obtain the same relative error. In the case of effective elastic moduli, the exact mean value and variance for a given domain size are a priori unknown. Using the equation (9.4), the absolute error on the mean value can be evaluated. This methodology was applied to the case of the dielectric permittivity of various random media $(5 ; 20)$, and to the elastic properties and thermal conductivity of a Voronoï mosaic (22), of materials from food industry (23), or of Boolean models of spheres (46).

9.3. The RVE of multiscale random media. The results in (46) indicate that the highest RVE sizes for the one-scale model (B) correspond to rigidly-reinforced media with $f \approx 0.7$. Accordingly, the variances $D_{Z}^{2}(V)$ and $D_{Z}^{2}$, with $Z=\sigma_{m}$, involved in equation 9.2 have been computed for the two-scales iterated Boolean model (IB) when $f_{1}=f_{2}=0.7$ and for a scale ratio equal to 10 . It is expected that the RVE sizes of porous or rigidly-reinforced multiscale media do not exceed the RVE size computed in this special case. One random sample of the (IB) model has been digitized on a volume of size $875^{3}$ voxels. The variances $D_{\sigma_{m}}^{2}(V)$ are then computed on subvolumes of the field $\sigma_{m}$ with $V=1, \ldots,(875 / 2)^{3}$. The results are shown for the case of quasi rigid inclusions in Fig. 10.7, where $D_{\sigma_{m}}^{2}(V)$ is plotted as a function of $V$ in $\log -\log$ scale. It is found that the integral range is about $A_{3}^{(I B)} \approx 5.110^{3}$, whereas $\sqrt{D_{\sigma_{m}}^{2}} \approx 4.410^{5}$. Accordingly, a $10 \%$ precision (i.e. $\epsilon=0.1$ ) is achieved when $V \approx 550^{3}$. Compared to the microstructure length scales, the size of the representative volume element at a $10 \%$ precision is about 1.7 times bigger than the diameter of the spheres at the largest scale and the integral range of the mean stress field is 5 times larger than that of the microstructure (IB). Finally, comparisons with similar results obtained in (46) for the one-scale Boolean model are as follow: at volume fraction $f=0.7$, the integral range for the iterated model is about 5.8 times greater than the integral range of the one-scale Boolean microstructure, whereas the fluctuations of the mean stress field $\sqrt{D_{\sigma_{m}}^{2}}$ are $\sim 32$ times greater. Equivalently, to achieve the same precision on the one-scale and iterated Boolean media, a volume of size 5.7 times larger is necessary for the latter.

10. Concluding remarks. This work presented a numerical study of the macroscopic behavior and local field distributions for special classes of multiscale composites with linear-elastic, conducting or insulating phases, with emphasis on infinitely contrasted media.

The coupling between 3D simulations of random sets and the numerical resolution of the equations of elasticity or of electrical current by means of FFT iterations is a powerful tool to estimate the effective properties of complex random media, even in the case of a high contrast between its components and for multiscale situations. 
Using this methodology, we could obtain the following main results: the incidence of the iteration of Boolean models with different scales is an increase of the effective properties with the number of scales for a given volume fraction of reinforcement particles (or alternatively a degradation of properties in the case of porous or insulating particles), as a result of the well-known lowering of the percolation threshold for an increasing number of scales (19). A separation of scale for the homogenization of properties was observed for one order of magnitude of the scales ratio. Using this principle, a prediction of the effective properties could be made by iteration. A preliminary application to the study of the percolation behaviour of such continuous microstructures enabled us to work out the non trivial evolution of some critical exponents close to the percolation threshold for the considered multiscale media. Finally, the statistical RVE was estimated in a two-scale example, and its size is found to be about 6 times larger than for the standard Boolean model at fixed precision.

Acknowledgements. This work was supported by Institut Carnot-Mines by a grant (Nanostructures project).

\section{References.}

[1] M. J. BerAn, AND J. MOLyneux, Use of classical variational principles to determine bounds for the effective bulk modulus in heterogeneous media, Quart. Appl. Math., 24 (1966), pp. 107-118.

[2] M. J. Beran, Statistical Continuum Theories, J. Wiley, New York, (1968).

[3] G. J. BerRyman, Variational bounds on elastic constants for the penetrable sphere model, J. Phys. D, 18 (1985): pp. 585-597.

[4] H.-S. CHEN, AND A. ACRIVOs, The effective elastic moduli of composite materials containing spherical inclusions at non-dilute concentrations, Int. J. Solids Structures 14, (1978) pp. 349-364.

[5] Delarue A., Prévision du comportement électromagnétique de matériaux composites à partir de leur mode d'élaboration et de leur morphologie, Thesis, Paris School of Mines (2001).

[6] W. T. Elam, A. R. Kerstein, And J. J. Rehr, Critical properties of the void percolation problem for spheres, Phys. Rev. Lett. 52, (1984), pp. 1516-1519.

[7] J. D. Eshelby, The determination of the elastic field of an ellipsoidal inclusion, and related problems, Proc. R. Soc. Lond. A 241 (1957), pp. 376-396.

[8] Z. Hashin, And S. Shtrikman, A variational approach to the theory of the effective magnetic permeability of multiphase materials, J. Appl. Phys. 33 (1962), pp. 3125-3131.

[9] Z. Hashin, And S. Shtrikman, A variational approach to the theory of the elastic behavior of multiphase materials, J. Mech. Phys. Solids 11 (1963), pp. 127 140.

[10] A. Jean, D. Jeulin, S. Cantournet, S. Forest, V. Mounoury, And F. N'GuYEN, Rubber with carbon black fillers: parameters identification of a multiscale nanostructure model, Proc. Eur. Conf. on Constitutive Models for Rubber ECCMR2007 (2007), Paris, September 4-7.

[11] D. Jeulin, Random structure analysis and modelling by Mathematical Morphology, Proc. CMDS5 ed. A. J. M. Spencer, Balkema, Rotterdam (1987) pp. 217-226.

[12] D. Jeulin, Modèles morphologiques de structures aléatoires et de changement d'échelle, Thèse de Doctorat d'État (1991), University of Caen.

[13] D. Jeulin, And A. LE CoËnt, Morphological modeling of random composites, Proc. CMDS8 Conference (Varna, 11-16 June 1995), ed K.Z. Markov, World Scientific Company (1996), pp. 199-206. 
[14] D. JEulin,. Modeling heterogeneous materials by random structures, Invited lecture, European Workshop on Application of Statistics and Probabilities in Wood Mechanics, Bordeaux. 22-23 March N-06/96/MM (1996), Paris School of Mines Publication.

[15] D. JEulin, Bounds of physical properties of some random structure, in: Proceedings of the CMDS9 Conference (Istanbul, Turkey, June 29-July 3, 1998), E. Inan \& K.Z. Markov (ed), World Scientific Publishing Company (1998), pp. 147-154.

[16] D. JEulin, Random Structure Models for Homogenization and Fracture Statistics, in: Mechanics of Random and Multiscale Microstructures, ed. by D. Jeulin and M. Ostoja-Starzewski, CISM Lecture Notes $N^{\circ} 430$, Springer Verlag, (2001), pp. 33-91.

[17] D. Jeulin, Random Structures in Physics, in: "Space, Structure and Randomness", Contributions in Honor of Georges Matheron in the Fields of Geostatistics, Random Sets, and Mathematical Morphology; Series: Lecture Notes in Statistics, Vol. 183, Bilodeau M., Meyer F., Schmitt M. eds. (2005), XIV, Springer-Verlag, pp. $183-222$.

[18] D. Jeulin, AND M. Moreaud, Multi-scale simulation of random spheres aggregates-Application to nanocomposites, Proc. of the 9th European Congress for Stereology, Zakopane (Poland), May 10-13 (2005), J. Chraponski, J. Cwajna, L. Wojnar eds.

[19] D. Jeulin, And M. Moreaud, Percolation d'agrégats multi-échelles de sphères et de fibres : Application aux nanocomposites, in: Matériaux (2006), Dijon, 13-17 nov.

[20] D. Jeulin, And M. Moreaud, Statistical representative volume element for predicting the dielectric permittivity of random media, Proc. CMDS 11, Paris, July 30-August 3 2007, D. Jeulin \& S. Forest eds. Les Presses de l'Ecole des Mines de Paris (2008), pp. 429-436.

[21] D. Jeulin, Morphological models of random structures, in preparation.

[22] T. Kanit, S. Forest, I. Galliet, V. Mounoury, and D. Jeulin, Determination of the size of the representative volume element for random composites: statistical and numerical approach, Int. J. of Solids and Structures 40 (2003), pp. 3647-3679.

[23] T. Kanit, F. N’Guyen, S. Forest, D. Jeulin, M. Reed, and S. SingleTON, Apparent and effective physical properties of heterogeneous materials: Representativity of samples of two materials from food industry, Comput. Methods Appl. Mech. Eng. 195 (2006), pp. 3960-3982.

[24] E. Kröner, Statistical Continuum Mechanics, Springer, Verlag (1971), Berlin.

[25] R. A. Lebensohn, Y. Liu, and P. Ponte Castañeda, On the accuracy of the self-consistent approximation for polycrystals: Comparisons with full-field numerical simulations, Acta Mat. 52 (2004), pp. 5347-5361.

[26] G. Matheron, Eléments pour une théorie des milieux poreux, Masson (1967), Paris.

[27] G. Matheron, Composition des perméabilités en milieu poreux hétérogène: critique de la règle de pondération géométrique, Rev. IFP 23 (1968), pp. 201-218.

[28] G. Matheron, The theory of regionalized variables and its applications, (1971) Paris School of Mines publications.

[29] G. Matheron, Random sets and integral geometry, (1975) J. Wiley, New York.

[30] G. Matheron, Estimating and Choosing, (1989) Springer Verlag, Berlin.

[31] J. J. MCCoy, On the deplacement field in an elastic medium with random vari- 
ations of material properties, Recent Advances in Engineering Sciences, Vol. 5 (1970), Gordon \& Breach, New York: pp. 235-254.

[32] J.-C. Michel, H. Moulinec, And P. Suquet, A computational scheme for linear and non-linear composites with arbitrary phase contrast, Int. J. Numer. Meth. Eng. 52 (2001), pp. 139-158.

[33] G. MiLton, Bounds on the elastic and transport properties of two component composites, J. Mech. Phys. Solids 30 (1982), pp. 177-191.

[34] H. Moulinec, And P. Suquet, A fast numerical method for computing the linear and nonlinear properties of composites, C. R. Acad. Sci. Paris II 318 (1994), pp. 1417-1423.

[35] H. Moulinec, And P. Suquet, A numerical method for computing the overall response of nonlinear composites with complex microstructure, Comp. Meth. Appl. Mech. Eng. 157 (1998), pp. 69-94.

[36] H. Moulinec, And P. Suquet, Intraphase strain heterogeneity in nonlinear composites: a computational approach, Eur. J. Mech. A 22 (2003), pp. 751-770.

[37] H. Moulinec, AND P. Suquet, Homogenization for nonlinear composites in the light of numerical simulations, in: Ponte Castañeda, P., et al. eds., Nonlinear Homogenization and its Applications to Composites, Polycrystals and Smart Materials, Kluwer Academic Publishers, The Netherlands (2004), pp. 193-223.

[38] H. Ottavi, J. Clerc, G. Giraud, J. Roussend, E. Guyon, and C. D. Mitescu, Electrical conductivity of a mixture of conducting and insulating spheres: an application of some percolation concepts, J. Phys. C: Solid State Phys., 11 (1978), pp. 1311-1328.

[39] I. Křivka, J. Prokeš, E. Tobolková, and J. Stejskal, Application of percolation concepts to electrical conductivity of polyaniline inorganic salt composites, J. of Mat. Chem., 10 (1999), pp. 2425-2428.

[40] M. D. Rintoul, And S. Torquato, Precise determination of the critical threshold and exponents in a three-dimensional continuum percolation model, J. Phys. A.: Math, 30 (1997), pp. 585-592.

[41] L. SAVARY, D. JEUlin AND A. ThOREL, Morphological analysis of carbonpolymer composite materials from thick sections, Acta Stereologica, 18, No.3 (1999), pp. 297-303.

[42] S. Torquato, And G. Stell, Microstructure of two-phase random media III. The n-point matrix probability functions for fully penetrable spheres, J. Chem. Phys., 79 (1983): pp. 1505-1510.

[43] S. Torquato, AND F. LAdo, Effective properties of two phase disordered composite media: II Evaluation of bounds on the conductivity and bulk modulus of dispersions of impenetrable spheres, Phys. Rev. B, 33 (1986): pp. 6428-6434.

[44] S. Torquato, Random heterogeneous media: microstructure and improved bounds on effective properties, Appl. Mech. Rev. 44 (1991): pp. 37-76.

[45] J. R. Willis, And J. R. ACton, The overall elastic moduli of a dilute suspension of spheres, The Quarterly J. of Mech. and App. Math. 29 (1976), pp. 163177.

[46] F. Willot, And D. Jeulin, Elastic behavior of composites containing Boolean random sets of inhomogeneities, Int. J. of Engng. Sc. 47 (2009), pp. 313-324. 


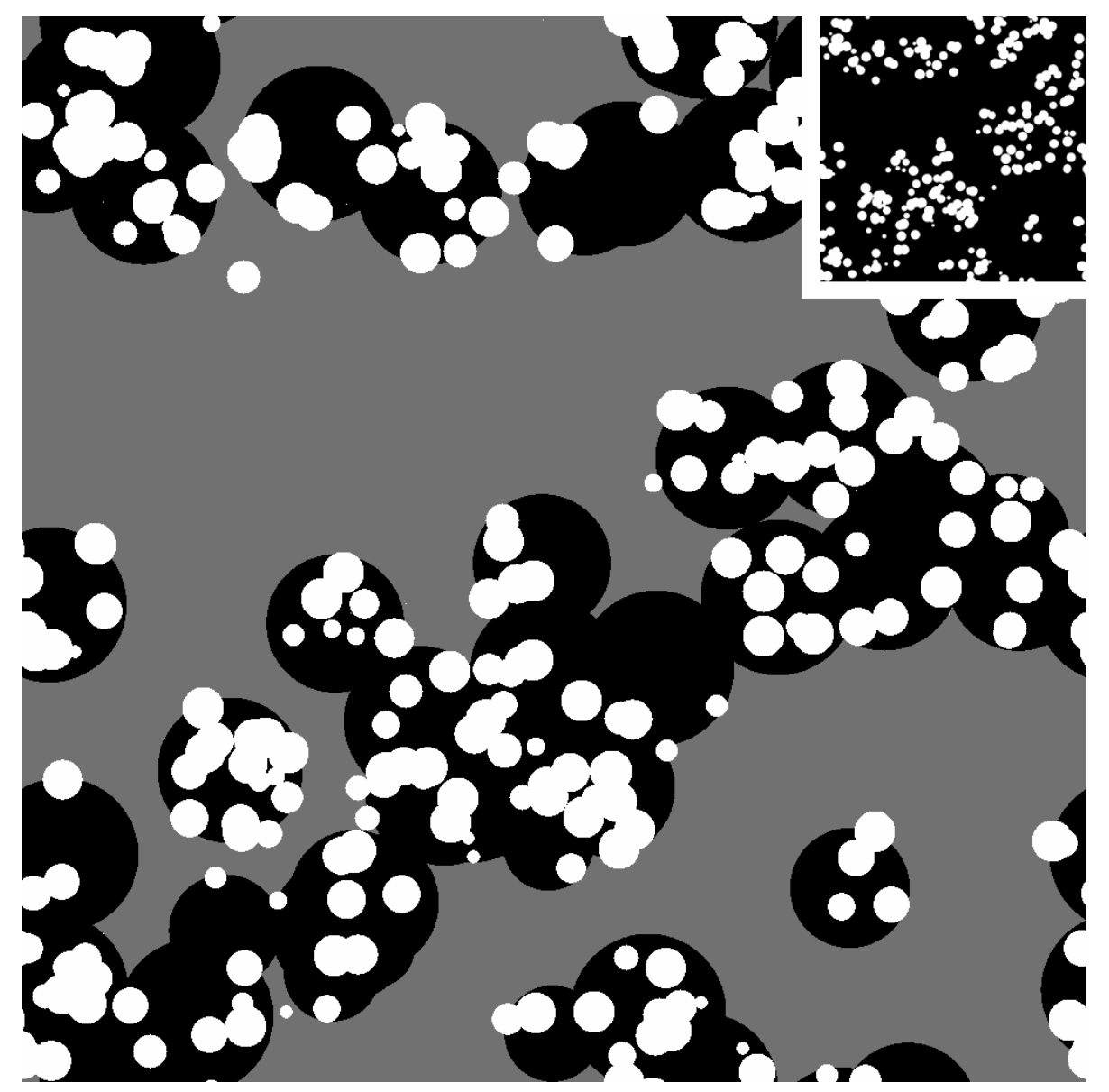

FIG. 10.1. 2D section of two embedded Boolean models (top-right, incrusted: final microstructure used in computations). 

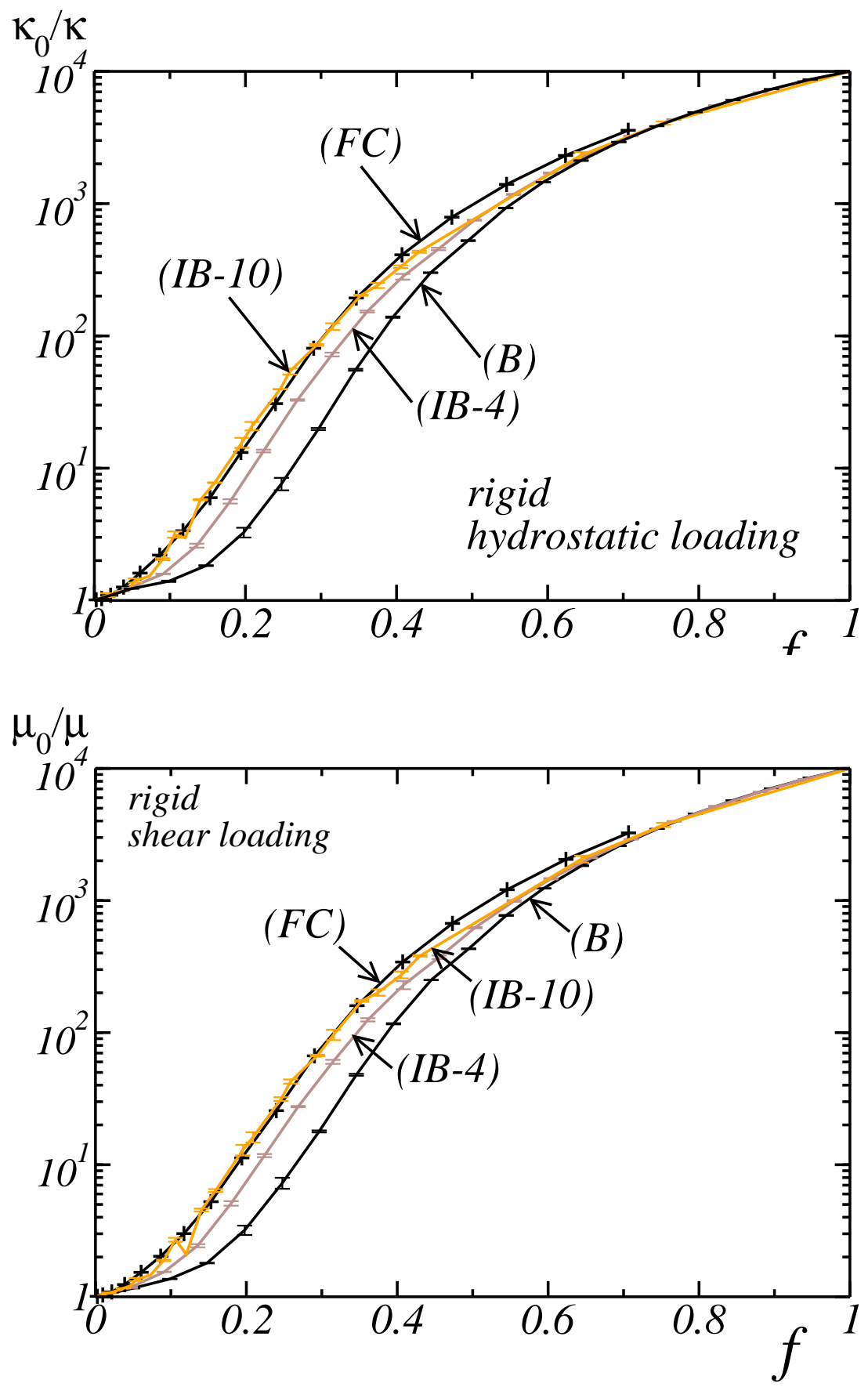

FIG. 10.2. Normalized effective bulk and shear moduli $\kappa_{0} / \kappa($ top $)$ and $\mu_{0} / \mu$ (bottom): comparison between two iterated Boolean medium with scales ratio $a^{(L)} / a=4,10$ (IB-4 and IB-10, resp.) and the equivalent one-scale finite-contrast model (FC). Values corresponding to the onescale Boolean medium (B) are shown for comparison. 

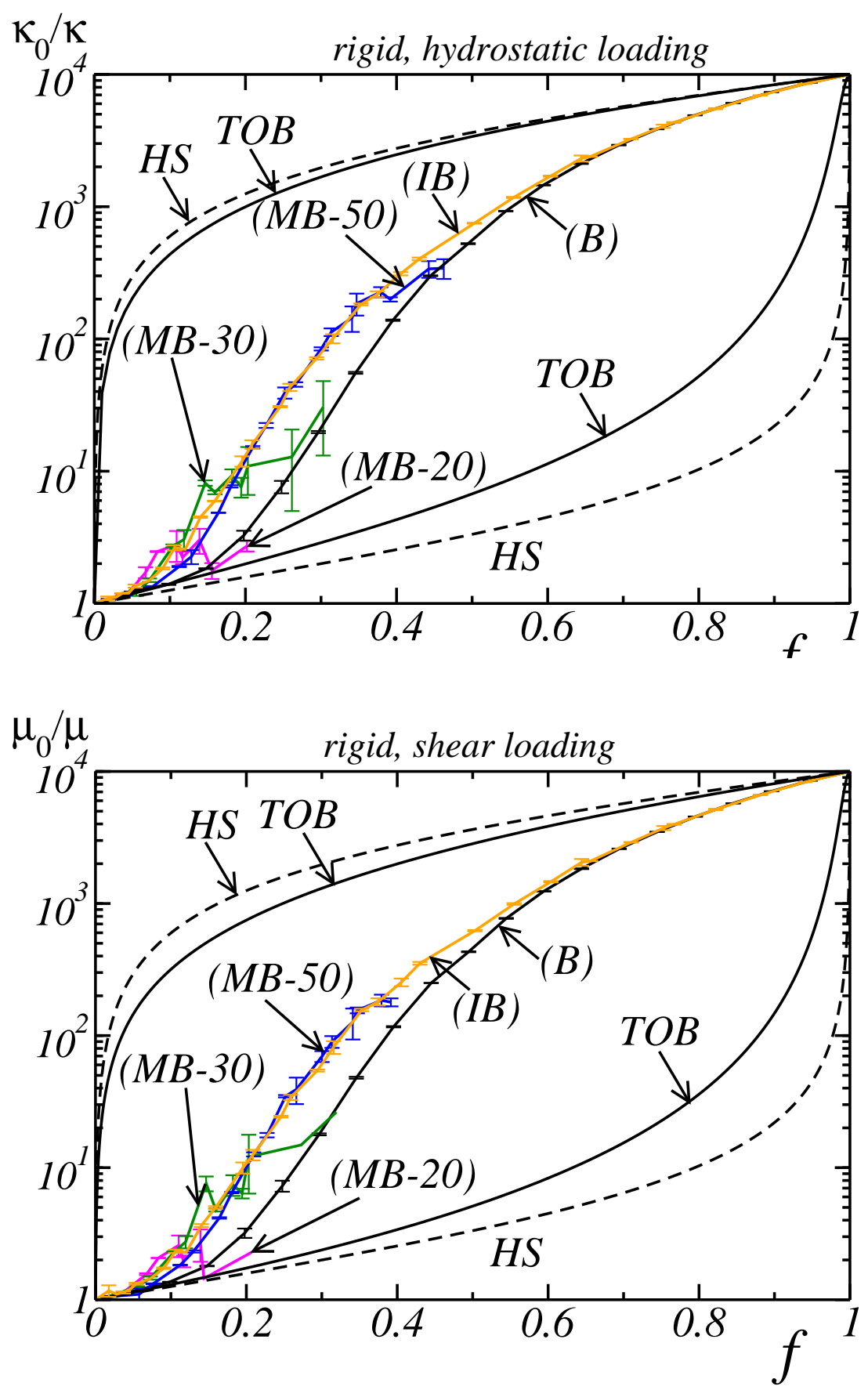

FIG. 10.3. Normalized effective bulk and shear moduli $\kappa_{0} / \kappa$ (top) and $\mu_{0} / \mu$ (bottom) as a function of the rigid inclusions volume fraction $f$, for various Boolean models: one-scale Boolean medium (B), two-scales iterated Boolean medium (IB), and several non-iterated two-scales Boolean media with $f^{(L)}=20 \%, 30 \%$ and $50 \%$ (MB-20, MB-30 and MB-50, resp.). The scales ratio for all multiscales media is a ${ }^{(L)} / a=10$. Hashin-Shtrikman (HS) and third-order Beran (TOB) bounds are provided for comparison. 

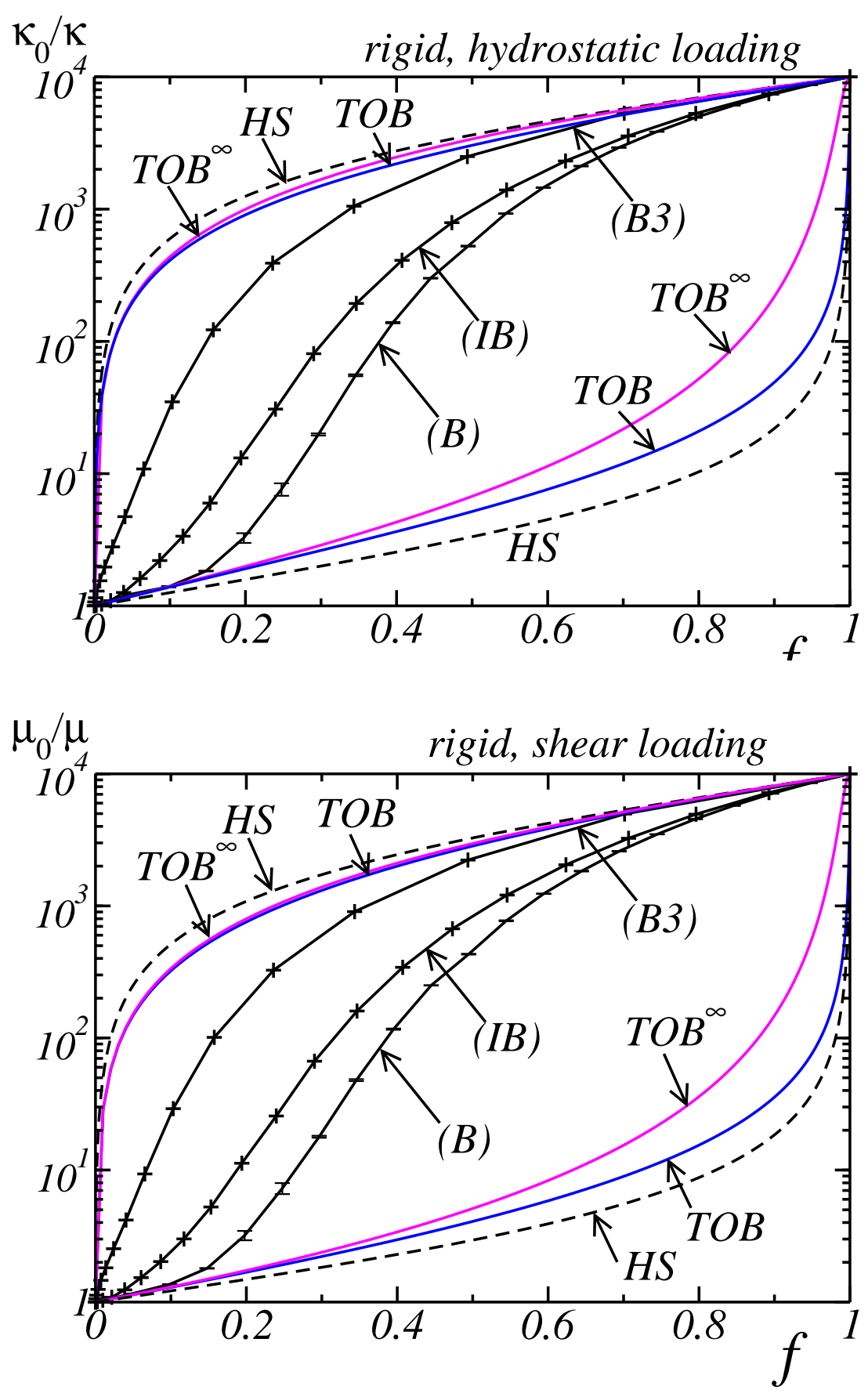

FIG. 10.4. FFT estimates and analytical bounds of the normalized bulk and shear moduli $\kappa_{0} / \kappa$ (top) and $\mu_{0} / \mu$ (bottom) as a functions of the volume fraction of rigid inclusions $f$, in the onescale model (B), the iterated two (IB) and three-scale (B3) Boolean microstructures. The dotted lines represent Hashikn and Shtrikman's lower and upper bounds (HS), whereas Beran's third-order bounds for the one-scale Boolean model and the Boolean model with infinitely many separated scales are shown as solid lines (TOB, TOB ${ }^{\infty}$, respectively). 

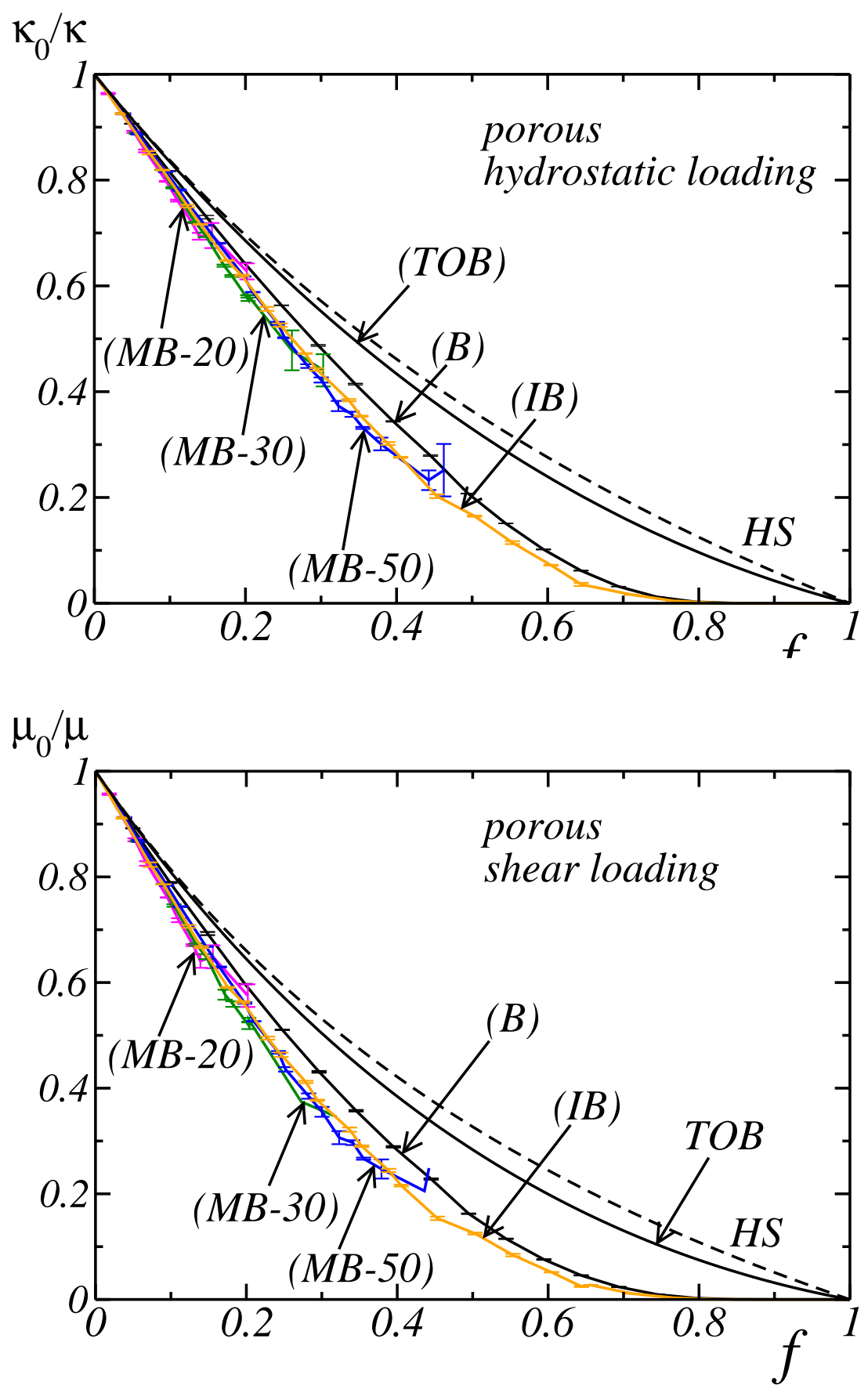

FIG. 10.5. Normalized effective bulk and shear moduli $\kappa_{0} / \kappa$ (top) and $\mu_{0} / \mu$ (bottom) as a function of porosity $f$, for various Boolean models: one-scale Boolean medium (B), two-scales iterated Boolean medium (IB), and several non-iterated two-scales Boolean media with $f^{(L)}=20 \%, 30 \%$ and $50 \%$ (MB-20, MB-30 and MB-50, resp.). The scales ratio for all multiscales media is $a^{(L)} / a=10$. Hashin-Shtrikman estimates (HS) are provided for comparison. 

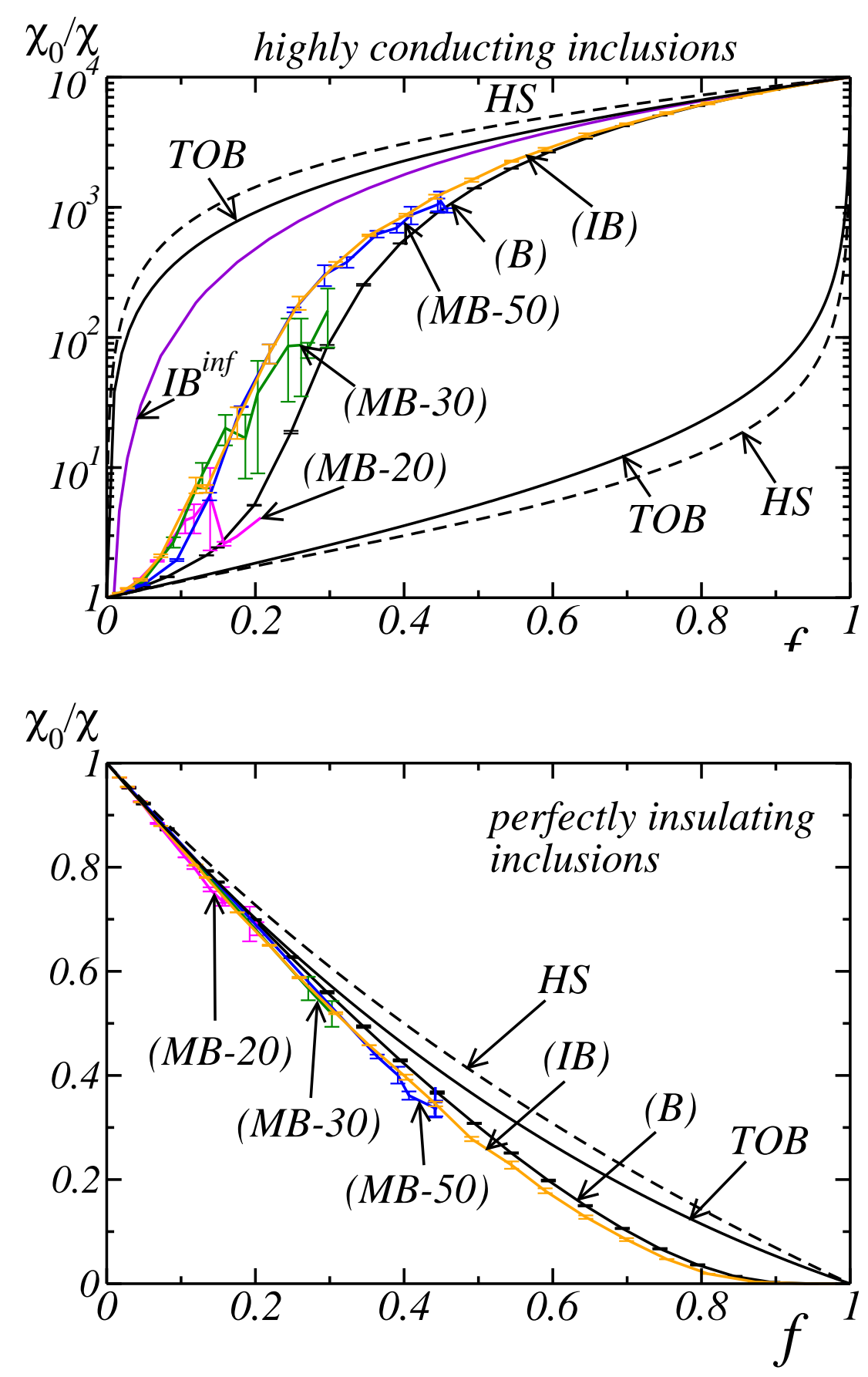

FIG. 10.6. Normalized effective conductivity $\chi_{0} / \chi$ as a function of the inclusion volume fractions $f$ (top: highly conduting inclusions; bottom: perfectly insulating inclusions), for various Boolean models: one-scale Boolean medium (B), two-scales iterated Boolean medium (IB), and several non-iterated two-scales Boolean media with $f^{(L)}=20 \%, 30 \%$ and $50 \%$ (MB-20, MB-30 and $M B-50$, resp.). The scales ratio for all multiscales media is $a^{(L)} / a=10$. Hashin-Shtrikman estimates (HS), Beran's third-order upper-bound (TOB) and an estimate for a material with an infinite number of scales ( $\mathrm{IB}^{\mathrm{inf}}$ ) are provided for comparison. 


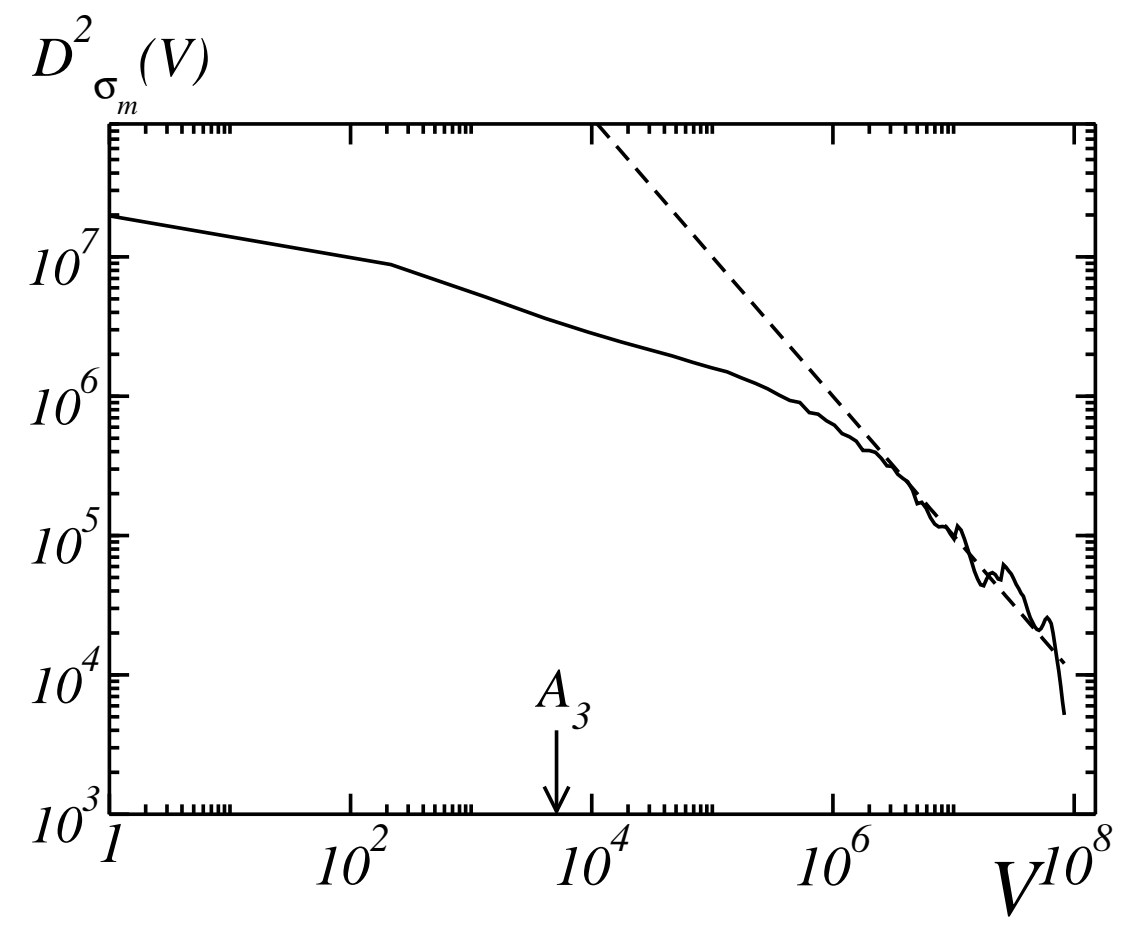

FIG. 10.7. Variance of the mean average stress field $\left\langle\sigma_{m}\right\rangle$, proportional to the material apparent bulk-modulus, as computed on volumes of size $V$, as a function of the volume size, for two-scales iterated Boolean model (IB) with quasi rigid inclusions, at $f=0.7$ (solid line). The dotted line represents a fit of the variance $D_{\sigma_{m}}^{2}(V)$ with Eq. 9.2, which holds for volumes much larger than the integral range, i.e. $V \gg A_{3}$. 


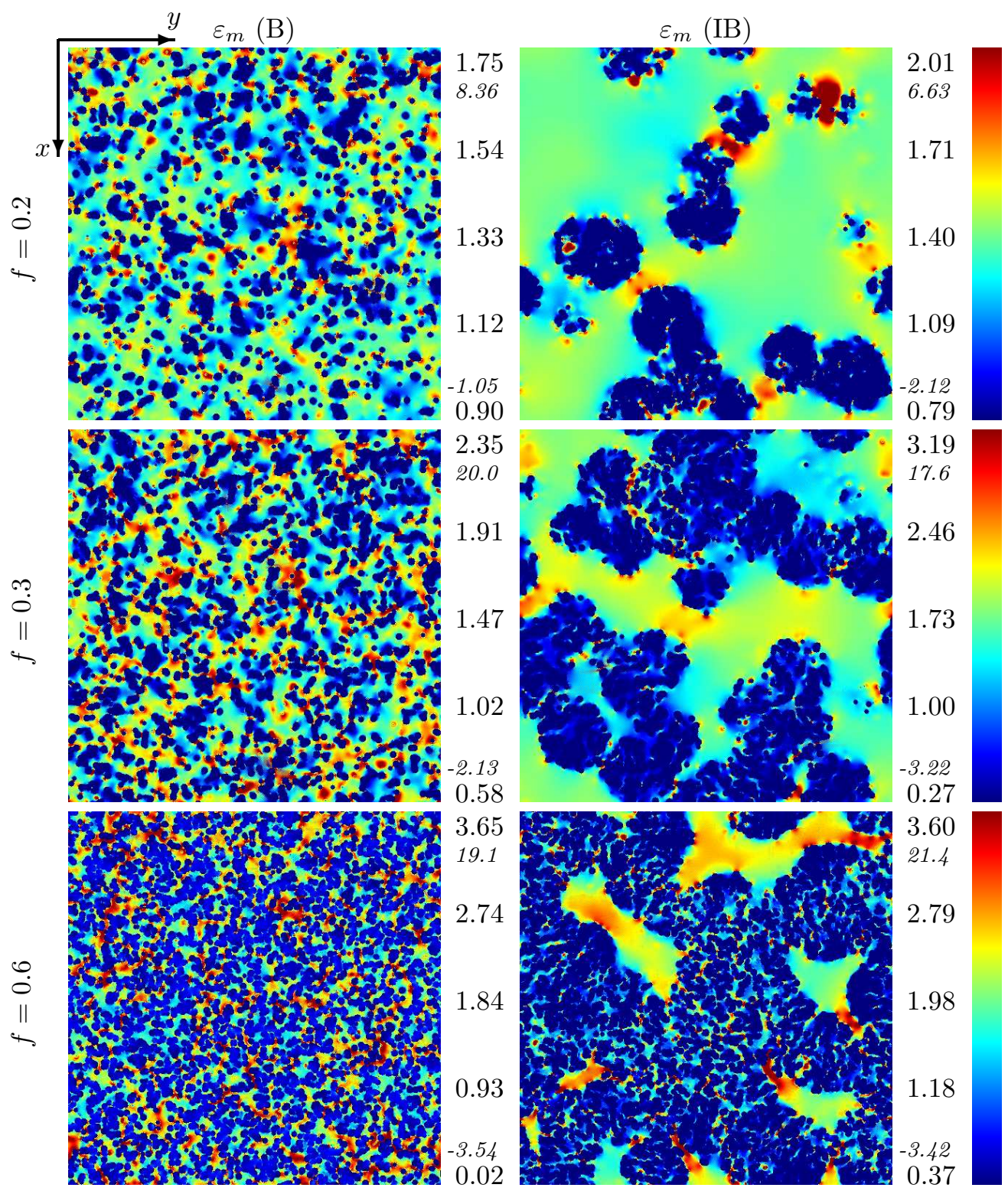

FIG. 10.8. $2 D$ sections in the $(x, y)$ plane of the mean strain field $\varepsilon_{m}$ in rigidly-reinforced onescale (B) and iterated two-scales (IB) Boolean models (left and right columns, resp.), at increasing sphere volume fractions $f=0.2,0.3,0.6$ (top to bottom). Hydrostatic strain loading is applied so that $\left\langle\varepsilon_{m}\right\rangle=1$. The field values are mapped with the color scale as indicated at the right of each map. To emphasize the fields patterns, $10 \%$ of the smallest and largest values are thresholded. The real minimum and maximum values are indicated in italic (colors online). 

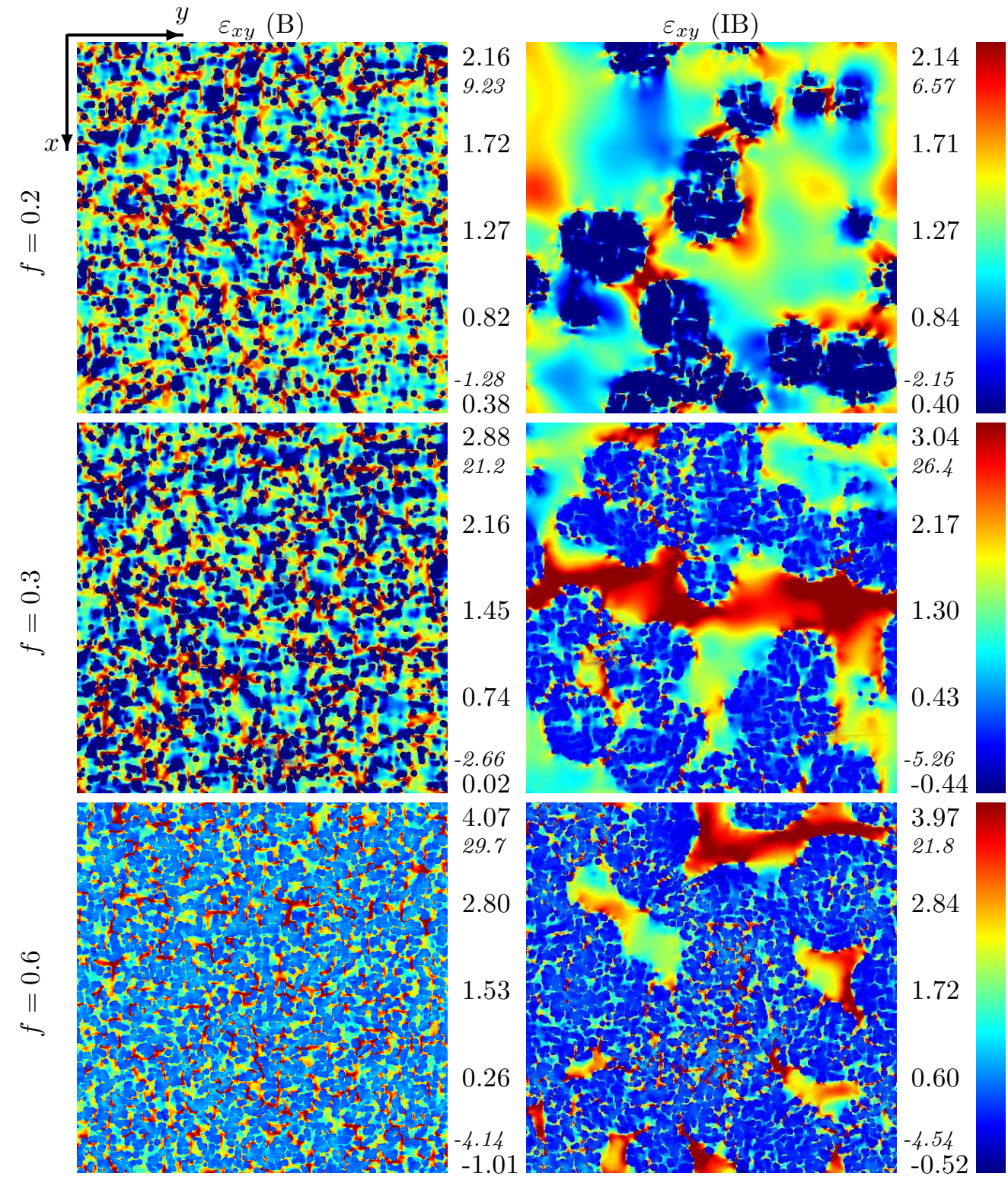

FIG. 10.9. Continuation of Fig. 10.8: component $\varepsilon_{x y}$, with applied strain shear loading $\left\langle\varepsilon_{x y}\right\rangle=1$. 


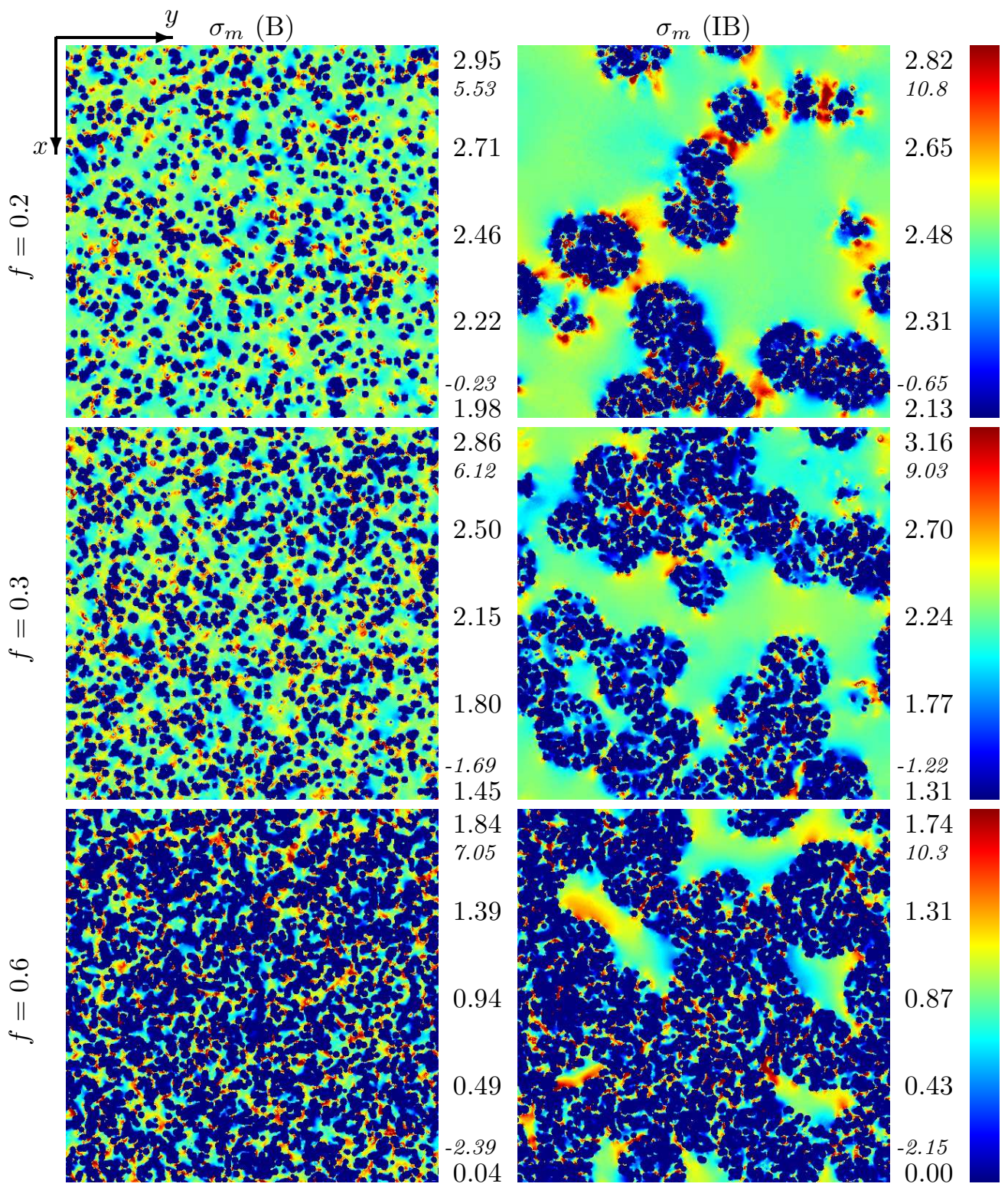

FIG. 10.10. $2 D$ sections in the $x, y$ plane of the mean stress field $\sigma_{m}$ in porous one-scale $(B)$ and iterated two-scales (IB) Boolean models (left and right columns, resp.), at increasing porosity $f=0.2,0.3,0.6$ (top to bottom). Hydrostatic strain loading is applied so that $\left\langle\varepsilon_{m}\right\rangle=1$. The field values are mapped with the color scale as indicated at the right of each map. To emphasize the fields patterns, $10 \%$ of the smallest and largest values are thresholded. The real minimum and maximum values are indicated in italic (colors online). 


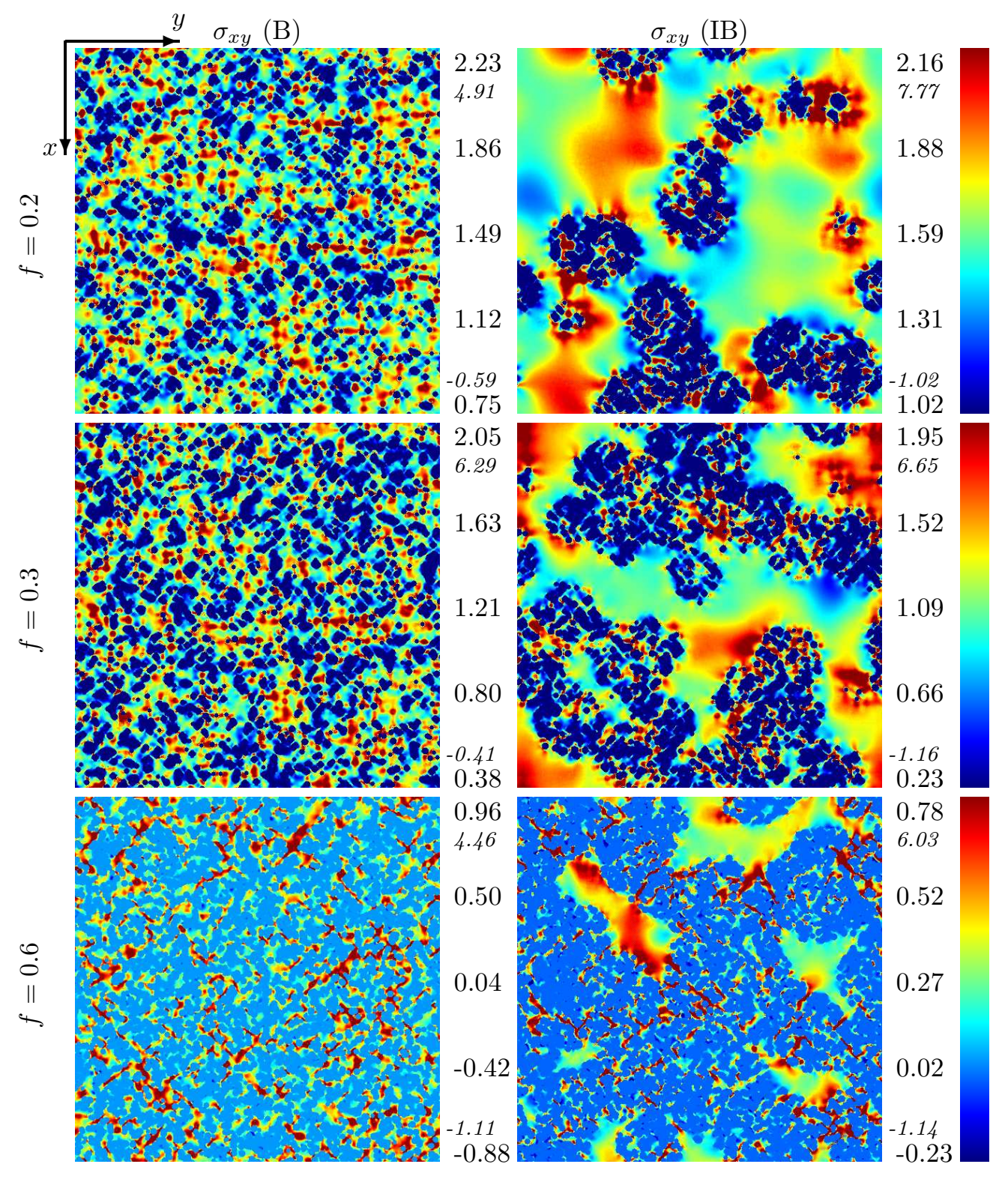

FIG. 10.11. Continuation of Fig. 10.10: component $\sigma_{x y}$, with applied strain shear loading $\left\langle\varepsilon_{x y}\right\rangle=1$. 